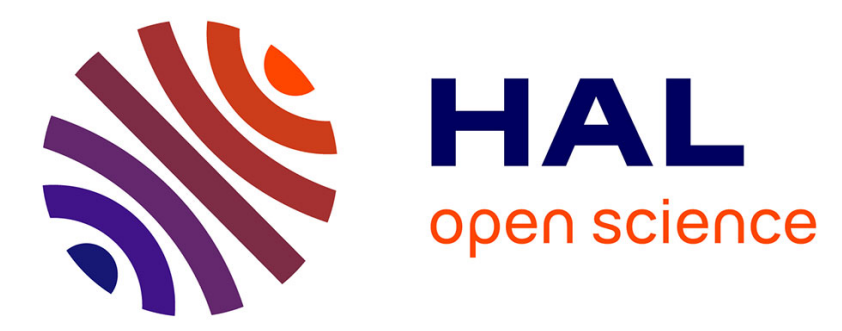

\title{
Étude de l'impact d'une formation sur la détection de la métaplasie intestinale gastrique en NBI \\ Victoria Grymonpré
}

\section{To cite this version:}

Victoria Grymonpré. Étude de l'impact d'une formation sur la détection de la métaplasie intestinale gastrique en NBI. Médecine humaine et pathologie. 2018. dumas-02972776

\section{HAL Id: dumas-02972776 \\ https://dumas.ccsd.cnrs.fr/dumas-02972776}

Submitted on 20 Oct 2020

HAL is a multi-disciplinary open access archive for the deposit and dissemination of scientific research documents, whether they are published or not. The documents may come from teaching and research institutions in France or abroad, or from public or private research centers.
L'archive ouverte pluridisciplinaire HAL, est destinée au dépôt et à la diffusion de documents scientifiques de niveau recherche, publiés ou non, émanant des établissements d'enseignement et de recherche français ou étrangers, des laboratoires publics ou privés.

\section{(1)(1) $\$(0)$}

Distributed under a Creative Commons Attribution - NonCommercial - ShareAlikel 4.0 


\section{UNIVERSITE DE MONTPELLIER FACULTE DE MEDECINE MONTPELLIER-NIMES}

\section{THESE}

Pour obtenir le titre de DOCTEUR EN MEDECINE

Présentée et soutenue publiquement

Par

Victoria GRYMONPRÉ

Le 25 octobre 2018

\section{Etude de l'impact d'une formation sur la détection de la métaplasie intestinale gastrique en NBI}

Directeur de thèse : Professeur Philippe POUDEROUX

JURY

Président :

Mr le Professeur Pierre BLANC

\section{Assesseurs :}

Mr le Professeur Éric ASSENAT

Mr le Professeur Philippe POUDEROUX

Mr le Docteur Jérôme HAZEBROUCQ 
UNIVERSITE DE MONTPELLIER FACULTE DE MEDECINE MONTPELLIER-NIMES

THESE

Pour obtenir le titre de DOCTEUR EN MEDECINE

Présentée et soutenue publiquement

Par

Victoria GRYMONPRÉ

Le 25 octobre 2018

\section{Etude de l'impact d'une formation sur la détection de la métaplasie intestinale gastrique en NBI}

Directeur de thèse : Professeur Philippe POUDEROUX

\section{JURY}

Président :

Mr le Professeur Pierre BLANC

\section{Assesseurs :}

Mr le Professeur Éric ASSENAT

Mr le Professeur Philippe POUDEROUX

Mr le Docteur Jérôme HAZEBROUCQ 
ANNEE UNIVERSITAIRE 2017 - 2018

PERSONNEL ENSEIGNANT

\section{Professeurs Honoraires}

ALLIEU Yves
ALRIC Robert
ARNAUD Bernard
ASTRUC Jacques
AUSSILLOUX Charles
AVEROUS Michel
AYRAL Guy
BAILLAT Xavier
BALDET Pierre
BALDY-MOULINIER Michel
BALMES Jean-Louis
BALMES Pierre
BANSARD Nicole
BAYLET René
BILLIARD Michel
BLARD Jean-Marie
BLAYAC Jean Pierre
BLOTMAN Francis
BONNEL François
BOUDET Charles
BOURGEOIS Jean-Marie
BRUEL Jean Michel
BUREAU Jean-Paul
BRUNEL Michel
CALLIS Albert
CANAUD Bernard
CASTELNAU Didier
CHAPTAL Paul-André
CIURANA Albert-Jean
CLOT Jacques
D'ATHIS Françoise
DEMAILLE Jacques
DESCOMPS Bernard
BARA

DIMEGLIO Alain
DU CAILAR Jacques
DUBOIS Jean Bernard
DUMAS Robert
DUMAZER Romain
ECHENNE Bernard
FABRE Serge
FREREBEAU Philippe
GALIFER René Benoît
GODLEWSKI Guilhem
GRASSET Daniel
GROLLEAU-RAOUX Robert
GUILHOU Jean-Jacques
HERTAULT Jean
HUMEAU Claude
JAFFIOL Claude
JANBON Charles
JANBON François
JARRY Daniel
JOYEUX Henri
LAFFARGUE François
LALLEMANT Jean Gabriel
LAMARQUE Jean-Louis
LAPEYRIE Henri
LESBROS Daniel
LOPEZ François Michel
LORIOT Jean
LOUBATIERES Marie
Madeleine
MAGNAN DE BORNIER
Bernard
MARY Henri
MATHIEU-DAUDE Pierre

\author{
MEYNADIER Jean \\ MICHEL François-Bernard \\ MICHEL Henri \\ MION Charles \\ MION Henri \\ MIRO Luis \\ NAVARRO Maurice \\ NAVRATIL Henri \\ OTHONIEL Jacques \\ PAGES Michel \\ PEGURET Claude \\ POUGET Régis \\ PUECH Paul \\ PUJOL Henri \\ PUJOL Rémy \\ RABISCHONG Pierre \\ RAMUZ Michel \\ RIEU Daniel \\ RIOUX Jean-Antoine \\ ROCHEFORT Henri \\ ROUANET DE VIGNE LAVIT \\ Jean Pierre \\ SAINT AUBERT Bernard \\ SANCHO-GARNIER Hélène \\ SANY Jacques \\ SENAC Jean-Paul \\ SERRE Arlette \\ SIMON Lucien \\ SOLASSOL Claude \\ THEVENET André \\ VIDAL Jacques \\ VISIER Jean Pierre
}


Professeurs Emérites

ARTUS Jean-Claude

BLANC François

BOULENGER Jean-Philippe

BOURREL Gérard

BRINGER Jacques

CLAUSTRES Mireille

DAURES Jean-Pierre

DAUZAT Michel

DEDET Jean-Pierre

ELEDJAM Jean-Jacques

GUERRIER Bernard

JOURDAN Jacques

MAURY Michèle

MILLAT Bertrand

MARES Pierre

MONNIER Louis

PRAT Dominique

PRATLONG Francine

PREFAUT Christian

PUJOL Rémy

ROSSI Michel

SULTAN Charles

TOUCHON Jacques

VOISIN Michel

ZANCA Michel 


\section{Professeurs des Universités - Praticiens Hospitaliers}

\section{PU-PH de classe exceptionnelle}

ALBAT Bernard - Chirurgie thoracique et cardiovasculaire

ALRIC Pierre - Chirurgie vasculaire ; médecine vasculaire (option chirurgie vasculaire)

BACCINO Eric - Médecine légale et droit de la santé

BASTIEN Patrick - Parasitologie et mycologie

BONAFE Alain - Radiologie et imagerie médicale

CAPDEVILA Xavier - Anesthésiologie-réanimation

COMBE Bernard - Rhumatologie

COSTA Pierre - Urologie

COTTALORDA Jérôme - Chirurgie infantile

COUBES Philippe - Neurochirurgie

CRAMPETTE Louis - Oto-rhino-laryngologie

CRISTOL Jean Paul - Biochimie et biologie moléculaire

DAVY Jean Marc - Cardiologie

DE LA COUSSAYE Jean Emmanuel - Anesthésiologie-réanimation

DELAPORTE Eric - Maladies infectieuses ; maladies tropicales

DE WAZIERES Benoît - Médecine interne ; gériatrie et biologie du vieillissement, médecine

générale, addictologie

DOMERGUE Jacques - Chirurgie générale

DUFFAU Hugues - Neurochirurgie

DUJOLS Pierre - Biostatistiques, informatique médicale et technologies de la communication

ELIAOU Jean François - Immunologie

FABRE Jean Michel - Chirurgie générale

GUILLOT Bernard - Dermato-vénéréologie

HAMAMAH Samir-Biologie et Médecine du développement et de la reproduction ;

gynécologie médicale

HEDON Bernard-Gynécologie-obstétrique ; gynécologie médicale

HERISSON Christian-Médecine physique et de réadaptation

JABER Samir-Anesthésiologie-réanimation

JEANDEL Claude-Médecine interne ; gériatrie et biologie du vieillissement, médecine générale, addictologie

JONQUET Olivier-Réanimation ; médecine d'urgence

JORGENSEN Christian-Thérapeutique ; médecine d'urgence ; addictologie

KOTZKI Pierre Olivier-Biophysique et médecine nucléaire

LANDAIS Paul-Epidémiologie, Economie de la santé et Prévention

LARREY Dominique-Gastroentérologie ; hépatologie ; addictologie

LEFRANT Jean-Yves-Anesthésiologie-réanimation

LE QUELLEC Alain-Médecine interne ; gériatrie et biologie du vieillissement, médecine générale, addictologie

MARTY-ANE Charles - Chirurgie thoracique et cardiovasculaire

MAUDELONDE Thierry - Biologie cellulaire

MERCIER Jacques - Physiologie

MESSNER Patrick - Cardiologie

MOURAD Georges-Néphrologie

PELISSIER Jacques-Médecine physique et de réadaptation

RENARD Eric-Endocrinologie, diabète et maladies métaboliques ; gynécologie médicale 
REYNES Jacques-Maladies infectieuses, maladies tropicales

RIBSTEIN Jean-Médecine interne ; gériatrie et biologie du vieillissement, médecine générale, addictologie

RIPART Jacques-Anesthésiologie-réanimation

ROUANET Philippe-Cancérologie ; radiothérapie

SCHVED Jean François-Hématologie; Transfusion

TAOUREL Patrice-Radiologie et imagerie médicale

UZIEL Alain -Oto-rhino-laryngologie

VANDE PERRE Philippe-Bactériologie-virologie ; hygiène hospitalière

YCHOU Marc-Cancérologie ; radiothérapie

\section{PU-PH de $1^{\text {re }}$ classe}

AGUILAR MARTINEZ Patricia-Hématologie ; transfusion

AVIGNON Antoine-Nutrition

AZRIA David -Cancérologie ; radiothérapie

BAGHDADLI Amaria-Pédopsychiatrie ; addictologie

BEREGI Jean-Paul-Radiologie et imagerie médicale

BLAIN Hubert-Médecine interne ; gériatrie et biologie du vieillissement, médecine

générale, addictologie

BLANC Pierre-Gastroentérologie ; hépatologie ; addictologie

BORIE Frédéric-Chirurgie digestive

BOULOT Pierre-Gynécologie-obstétrique ; gynécologie médicale

CAMBONIE Gilles -Pédiatrie

CAMU William-Neurologie

CANOVAS François-Anatomie

CARTRON Guillaume-Hématologie ; transfusion

CHAMMAS Michel-Chirurgie orthopédique et traumatologique

COLSON Pascal-Anesthésiologie-réanimation

CORBEAU Pierre-Immunologie

COSTES Valérie-Anatomie et cytologie pathologiques

COURTET Philippe-Psychiatrie d'adultes ; addictologie

CYTEVAL Catherine-Radiologie et imagerie médicale

DADURE Christophe-Anesthésiologie-réanimation

DAUVILLIERS Yves-Physiologie

DE TAYRAC Renaud-Gynécologie-obstétrique, gynécologie médicale

DEMARIA Roland-Chirurgie thoracique et cardio-vasculaire

DEMOLY Pascal-Pneumologie ; addictologie

DEREURE Olivier-Dermatologie - vénéréologie

DROUPY Stéphane -Urologie

DUCROS Anne-Neurologie -

FRAPIER Jean-Marc-Chirurgie thoracique et cardiovasculaire

KLOUCHE Kada-Réanimation ; médecine d'urgence

KOENIG Michel-Génétique moléculaire

LABAUGE Pierre- Neurologie

LAFFONT Isabelle-Médecine physique et de réadaptation

LAVABRE-BERTRAND Thierry-Cytologie et histologie

LECLERCQ Florence-Cardiologie

LEHMANN Sylvain-Biochimie et biologie moléculaire

LUMBROSO Serge-Biochimie et Biologie moléculaire 
MARIANO-GOULART Denis-Biophysique et médecine nucléaire

MATECKI Stéfan -Physiologie

MEUNIER Laurent-Dermato-vénéréologie

MONDAIN Michel-Oto-rhino-laryngologie

MORIN Denis-Pédiatrie

NAVARRO Francis-Chirurgie générale

PAGEAUX Georges-Philippe-Gastroentérologie ; hépatologie ; addictologie

PETIT Pierre-Pharmacologie fondamentale; pharmacologie clinique ; addictologie

PERNEY Pascal-Médecine interne ; gériatrie et biologie du vieillissement, médecine générale, addictologie

PUJOL Jean Louis-Pneumologie ; addictologie

PUJOL Pascal-Biologie cellulaire

PURPER-OUAKIL Diane-Pédopsychiatrie ; addictologie

QUERE Isabelle-Chirurgie vasculaire ; médecine vasculaire (option médecine vasculaire)

SOTTO Albert-Maladies infectieuses; maladies tropicales

TOUITOU Isabelle-Génétique

TRAN Tu-Anh-Pédiatrie

VERNHET Hélène-Radiologie et imagerie médicale

\section{PU-PH de 2ème classe}

ASSENAT Éric-Gastroentérologie ; hépatologie ; addictologie

BERTHET Jean-Philippe-Chirurgie thoracique et cardiovasculaire

BOURDIN Arnaud-Pneumologie ; addictologie

CANAUD Ludovic-Chirurgie vasculaire ; Médecine Vasculaire

CAPDEVIELLE Delphine-Psychiatrie d'Adultes; addictologie

CAPTIER Guillaume-Anatomie

CAYLA Guillaume-Cardiologie

CHANQUES Gérald-Anesthésiologie-réanimation

COLOMBO Pierre-Emmanuel-Cancérologie ; radiothérapie

COSTALAT Vincent-Radiologie et imagerie médicale

COULET Bertrand-Chirurgie orthopédique et traumatologique

CUVILLON Philippe-Anesthésiologie-réanimation

DAIEN Vincent-Ophtalmologie

DE VOS John-Cytologie et histologie

DORANDEU Anne-Médecine légale -

DUPEYRON Arnaud-Médecine physique et de réadaptation

FESLER Pierre-Médecine interne ; gériatrie et biologie du vieillissement, médecine générale, addictologie

GARREL Renaud -Oto-rhino-laryngologie

GAUJOUX Viala Cécile-Rhumatologie

GENEVIEVE David-Génétique

GODREUIL Sylvain-Bactériologie-virologie ; hygiène hospitalière

GUILLAUME Sébastien-Urgences et Post urgences psychiatriques -

GUILPAIN Philippe-Médecine Interne, gériatrie et biologie du vieillissement; addictologie

GUIU Boris-Radiologie et imagerie médicale

HAYOT Maurice-Physiologie

HOUEDE Nadine-Cancérologie ; radiothérapie

JACOT William-Cancérologie ; Radiothérapie

JUNG Boris-Réanimation ; médecine d'urgence 
KALFA Nicolas-Chirurgie infantile

KOUYOUMDJIAN Pascal-Chirurgie orthopédique et traumatologique

LACHAUD Laurence-Parasitologie et mycologie

LALLEMANT Benjamin-Oto-rhino-laryngologie

LAVIGNE Jean-Philippe-Bactériologie-virologie ; hygiène hospitalière

LE MOING Vincent-Maladies infectieuses; maladies tropicales

LETOUZEY Vincent-Gynécologie-obstétrique ; gynécologie médicale

LOPEZ CASTROMAN Jorge-Psychiatrie d'Adultes; addictologie

LUKAS Cédric-Rhumatologie

MAURY Philippe-Chirurgie orthopédique et traumatologique

MILLET Ingrid-Radiologie et imagerie médicale

MORANNE Olvier-Néphrologie

MOREL Jacques -Rhumatologie

NAGOT Nicolas-Biostatistiques, informatique médicale et technologies de la communication

NOCCA David-Chirurgie digestive

PANARO Fabrizio-Chirurgie générale

PARIS Françoise-Biologie et médecine du développement et de la reproduction ; gynécologie médicale

PASQUIE Jean-Luc-Cardiologie

PEREZ MARTIN Antonia-Physiologie

POUDEROUX Philippe-Gastroentérologie ; hépatologie ; addictologie

PRUDHOMME Michel-Anatomie

RIGAU Valérie-Anatomie et cytologie pathologiques

RIVIER François-Pédiatrie

ROGER Pascal-Anatomie et cytologie pathologiques

ROSSI Jean François-Hématologie ; transfusion

ROUBILLE François-Cardiologie

SEBBANE Mustapha-Anesthésiologie-réanimation

SEGNARBIEUX François-Neurochirurgie

SIRVENT Nicolas-Pédiatrie

SOLASSOL Jérôme-Biologie cellulaire

SULTAN Ariane-Nutrition

THOUVENOT Éric-Neurologie

THURET Rodolphe-Urologie

VENAIL Frédéric-Oto-rhino-laryngologie

VILLAIN Max-Ophtalmologie

VINCENT Denis -Médecine interne ; gériatrie et biologie du vieillissement, médecine générale, addictologie

VINCENT Thierry-Immunologie

WOJTUSCISZYN Anne-Endocrinologie-diabétologie-nutrition

\section{PROFESSEURS DES UNIVERSITES}

$\underline{1^{\text {re }} \text { classe : }}$

COLINGE Jacques - Cancérologie, Signalisation cellulaire et systèmes complexes

$2^{\text {ème }}$ classe :

LAOUDJ CHENIVESSE Dalila - Biochimie et biologie moléculaire

VISIER Laurent - Sociologie, démographie 
PROFESSEURS DES UNIVERSITES - Médecine générale

$\underline{1^{\text {re }} \text { classe : }}$

LAMBERT Philippe

$2^{\text {ème }}$ classe :

AMOUYAL Michel

DAVID Michel

PROFESSEURS ASSOCIES - Médecine Générale

RAMBAUD Jacques

\section{PROFESSEUR ASSOCIE - Médecine}

BESSIS Didier - Dermato-vénéréologie)

PERRIGAULT Pierre-François - Anesthésiologie-réanimation; médecine d'urgence

ROUBERTIE Agathe - Pédiatrie

\section{Maîtres de Conférences des Universités - Praticiens Hospitaliers}

\section{MCU-PH Hors classe}

CACHEUX-RATABOUL Valère-Génétique

CARRIERE Christian-Bactériologie-virologie ; hygiène hospitalière

CHARACHON Sylvie-Bactériologie-virologie ; hygiène hospitalière

FABBRO-PERAY Pascale-Epidémiologie, économie de la santé et prévention

HILLAIRE-BUYS Dominique-Pharmacologie fondamentale; pharmacologie clinique ; addictologie PELLESTOR Franck-Cytologie et histologie

PUJOL Joseph-Anatomie

RAMOS Jeanne-Anatomie et cytologie pathologiques

RICHARD Bruno-Thérapeutique ; addictologie

RISPAIL Philippe-Parasitologie et mycologie

SEGONDY Michel-Bactériologie-virologie ; hygiène hospitalière

STOEBNER Pierre -Dermato-vénéréologie

\section{MCU-PH de $1^{\text {re }}$ classe}

ALLARDET-SERVENT Annick-Bactériologie-virologie ; hygiène hospitalière

BADIOU Stéphanie-Biochimie et biologie moléculaire

BOUDOUSQ Vincent-Biophysique et médecine nucléaire

BOULLE Nathalie-Biologie cellulaire

BOURGIER Céline-Cancérologie ; Radiothérapie

BRET Caroline -Hématologie biologique

COSSEE Mireille-Génétique Moléculaire

GABELLE DELOUSTAL Audrey-Neurologie

GIANSILY-BLAIZOT Muriel-Hématologie ; transfusion

GIRARDET-BESSIS Anne-Biochimie et biologie moléculaire

LAVIGNE Géraldine-Hématologie ; transfusion

LE QUINTREC Moglie-Néphrologie

MATHIEU Olivier-Pharmacologie fondamentale ; pharmacologie clinique ; addictologie

MENJOT de CHAMPFLEUR Nicolas-Neuroradiologie 
MOUZAT Kévin-Biochimie et biologie moléculaire

PANABIERES Catherine-Biologie cellulaire

PHILIBERT Pascal-Biologie et médecine du développement et de la reproduction

RAVEL Christophe - Parasitologie et mycologie

SCHUSTER-BECK Iris-Physiologie

STERKERS Yvon-Parasitologie et mycologie

TUAILLON Edouard-Bactériologie-virologie ; hygiène hospitalière

YACHOUH Jacques-Chirurgie maxillo-faciale et stomatologie

MCU-PH de $2^{\text {éme }}$ classe

BERTRAND Martin-Anatomie

BRUN Michel-Bactériologie-virologie; hygiène hospitalière

DU THANH Aurélie-Dermato-vénéréologie

GALANAUD Jean Philippe-Médecine Vasculaire

GOUZI Farès-Physiologie

JEZIORSKI Éric-Pédiatrie

KUSTER Nils-Biochimie et biologie moléculaire

LESAGE François-Xavier-Médecine et Santé au Travail

MAKINSON Alain-Maladies infectieuses, Maladies tropicales

MURA Thibault-Biostatistiques, informatique médicale et technologies de la communication

OLIE Emilie-Psychiatrie d'adultes; addictologie

THEVENIN-RENE Céline-Immunologie

\section{MAITRES DE CONFERENCES DES UNIVERSITES - Médecine Générale}

COSTA David

FOLCO-LOGNOS Béatrice

\section{MAITRES DE CONFERENCES ASSOCIES - Médecine Générale}

CLARY Bernard

GARCIA Marc

MILLION Elodie

PAVAGEAU Sylvain

REBOUL Marie-Catherine

SEGURET Pierre

\section{MAITRES DE CONFERENCES DES UNIVERSITES}

Maîtres de Conférences hors classe

BADIA Eric - Sciences biologiques fondamentales et cliniques

\section{Maîtres de Conférences de classe normale}

BECAMEL Carine - Neurosciences

BERNEX Florence - Physiologie

CHAUMONT-DUBEL Séverine - Sciences du médicament et des autres produits de santé

CHAZAL Nathalie - Biologie cellulaire

DELABY Constance - Biochimie et biologie moléculaire

GUGLIELMI Laurence - Sciences biologiques fondamentales et cliniques

HENRY Laurent - Sciences biologiques fondamentales et cliniques

LADRET Véronique - Mathématiques appliquées et applications des mathématiques 
LAINE Sébastien - Sciences du Médicament et autres produits de santé LE GALLIC Lionel - Sciences du médicament et autres produits de santé LOZZA Catherine - Sciences physico-chimiques et technologies pharmaceutiques MAIMOUN Laurent - Sciences physico-chimiques et ingénierie appliquée à la santé MOREAUX Jérôme - Science biologiques, fondamentales et cliniques MORITZ-GASSER Sylvie - Neurosciences

MOUTOT Gilles - Philosophie PASSERIEUX Emilie - Physiologie

RAMIREZ Jean-Marie - Histologie

TAULAN Magali - Biologie Cellulaire

\section{PRATICIENS HOSPITALIERS UNIVERSITAIRES}

\section{CLAIRE DAIEN-Rhumatologie}

BASTIDE Sophie-Epidémiologie, économie de la santé et prévention

FAILLIE Jean-Luc-

Pharmacologie fondamentale ; pharmacologie clinique ; addictologie

GATINOIS Vincent-Histologie, embryologie et cytogénétique

HERLIN Christian -Chirurgie plastique ; reconstructrice et esthétique ; brûlologie

HERRERO Astrid-Chirurgie générale

PANTEL Alix-Bactériologie-virologie ; hygiène hospitalière

PERS Yves-Marie-Thérapeutique, médecine d'urgence; addictologie

PINETON DE CHAMBRUN Guillaume-Gastroentérologie ; hépatologie ; addictologie TORRE Antoine-Gynécologie-obstétrique ; gynécologie médicale 


\section{REMERCIEMENTS:}

\section{A Monsieur le Professeur Philippe Pouderoux,}

Pour être à l'initiative de ce projet que j'ai eu beaucoup de plaisir à réaliser, pour votre passion pour l'endoscopie qui m'a permis d'approfondir mes connaissances, et pour le temps passé à réfléchir et progresser ensemble sur ce travail.

\section{A Monsieur le Professeur Pierre Blanc,}

Pour m'avoir transmis la rigueur dans mon travail quotidien, pour votre honnêteté. Merci également de m'avoir permis de réaliser les projets qui me tenaient à cœur et de m'accorder votre confiance pour mes futures fonctions.

\section{A Monsieur le Professeur Éric Assenat,}

Pour avoir pris le temps de participer à ce travail et pour avoir accepté d'être dans ce jury. Merci pour les belles connaissances que vous nous transmettez en oncologie digestive.

\section{A Monsieur le Docteur Jérôme Hazebroucq,}

Pour le temps passé sur ce travail qui m'as permis de beaucoup mieux comprendre le lien entre endoscopie et anatomie pathologique, merci également pour ta gentillesse et ton soutien. 
Au Docteur Guillaume Pineton de Chambrun, pour la transmission de tes connaissances, particulièrement dans les $\mathrm{MICl}$, et ton investissement dans la recherche qui me donne envie de faire avancer la médecine.

Aux Docteurs Michael Bismuth et Jean Christophe Valats, pour la transmission de vos connaissances et votre gentillesse. Je suis heureuse de bientôt rejoindre votre équipe.

Aux Docteurs Gaspard Dufour, Roman Combes, Marie Dupuy et Jose Ursic-Bedoya qui m'ont accompagné, soutenu et avec qui j'ai partagé de bons moments tout au long de cet internat.

A l'équipe de Nîmes, les Docteurs Jean-François Bourgaux, Ludovic Caillo, Camille Bories, Valérie Phoutthasang et Bénédicte Brunaud-Gagniard pour votre participation à l'étude, votre gentillesse et votre esprit d'équipe.

A l'équipe de nutrition de l'ICM :

Le Docteur Pierre Senesse, pour ta générosité, ta gentillesse et ton humour qui permettent de détendre toutes les situations. Merci pour ton écoute et ton soutien. Le Docteur Nicolas Flori, pour la transmission de ta rigueur et tes conseils avisés qui m'ont permis de bien progresser en endoscopie.

Le Docteur Héloïse Lecornu, pour ta patience et ta gentillesse.

A Arnaud, Anne, Laure, Steph, Marie, Cathy pour votre bonne humeur et votre soutien durant ce semestre, merci de m'avoir transmis vos connaissances en nutrition.

A mes co-internes, Barbara, Ardoudou, Najiji, Nico, Franzouille, Claire, Anne pour tous ces moments passés ensemble en stage et en dehors qui nous ont rapproché. On se retrouve bientôt! 
A ma Maman, pour ton soutien, ton amour et ton sens de l'organisation qui m'ont toujours beaucoup aidé de la première jusqu'à la dernière année. Je te dois énormément dans cette réussite, merci. Je t'aime fort.

A mon père, pour ton amour même si nous ne sommes pas en accord sur tout.

A Vincent, Beau-papa, pour ces longues discussions qui ont participé à me faire choisir la médecine, pour ton soutien tout au long de ces études, et pour l'ambiance musicale qui a aiguayé mes révisions.

A Mattéo, mon petit frère maintenant plus grand moi ;) pour la joie de te voir courir vers moi chaque week-end quand je rentrais, pour ton soutien ces derniers mois qui a beaucoup compté pour moi.

A mes grands-parents, pour votre amour, votre présence qui m'ont aidé à progresser jusque-là. Je vous aime fort.

A Tonton et Anne-So, toujours présents pour m'aider à prendre les décisions importantes, et fêter les évènements importants de vie. Merci pour votre soutien, vous comptez énormément.

A ma Marraine, je sais que tu seras toujours là pour moi. Je me souviendrais toujours de ton ordonnance en $1^{\text {ère }}$ année de médecine..! Elle m'a aidé à bien démarrer mes études ;) 
A Marie (ma Chou), pour toutes les valeurs de vie que l'on partage, pour toutes ces heures en amphi qui sont devenues de bons moments en grande partie grâce à toi, pour ta fidélité en amitié. <3

A Lauriane (Laulau), pour tous ces fous rires et délires de l'externat, pour avoir su revenir vers moi et pour cette belle amitié pas prête de s'arrêter.

A Charlotte (ma Cha), pour tous nos délires et fêtes de l'externat et de la marine marchande, pour avoir supporté toutes nos discussions de médecine, pour avoir su préserver notre belle amitié malgré la distance.

A mes amies et colocs de cœur qui m'ont permis de passer deux fabuleuses années de mon internat :

Mélissa (ma coquillette), pour notre complicité sans limite, pour toujours me suivre dans les idées les plus saugrenues, pour ton énorme soutien ces derniers mois et pour toujours avoir les bons mots aux bons moments.. Tu sais déjà le reste ;)

Marion (chouchou), pour notre Jean Cuculles qui me fera toujours mourir de rire, pour ta sensibilité et ta gentillesse, pour toutes ces soirées de fête ou de plaids toujours aussi géniales.

Claire (Clairou), pour ta bonne humeur quotidienne, pour être toujours partante pour un mojito et toujours avoir ce qu'il faut dans ton sac;) ces 2 ans de coloc m'ont vraiment fait découvrir une belle personne, une amie.

A Julia, beaucoup de $\mathrm{km}$ nous séparent mais notre amitié et notre complicité de bougent pas d'un poil depuis tout ce temps. Déjà hâte que l'on reparte à l'aventure ensemble!

A Céline, pou avoir enduré la P1 ensemble et on sait que c'est une épreuve, pour ces beaux voyages que l'on a fait.. Et j'espère que ça ne s'arrêtera pas là ! Pour une amitié qui dure. Jo je pense également à toi pour la P1.. et tout le reste ;) 
A Antonia, pour avoir franchi le pas de l'externat vers l'internat ensemble, pour ta motivation, pour ton grand soutien ces derniers mois <3 Une pensée également pour toi Dèb!

A tous ceux avec qui j'ai partagé de beaux moments durant l'externat : Sandrine, Mathieu, Guillaume, Victorine, Chris, Laura, Mélanie, Camille, Elsa, Mika.. Je pense fort à vous!

A ma famille Biteroise sans qui l'internat n'aurait pas été le même, Robinou pour tes petites attentions touchantes, Chloé, Marie, Ninou, Aude, Philine et tous les autres, pour ces 6 premiers mois de folie ensemble nous ont soudé pour partager encore plus après.

A la team St Eloi, Babar (Comme un rossignol.. je l'entends encore haha), Tinou, Bobo, Nath pour ces covoit de folie, Sarah, Guigui, Susu.. et tous les autres, pour tous ces moments partagés de fête et de rire qui ont égayé mon internat. 
ABREVIATIONS:

NBI : Narrow-Band Imaging

OLGA : Operative Link for Gastritis Assessment

OLGIM : Operative Link on Intestinal Metaplasia

HP : Helicobacter Pylori

WOS : White Opaque Substance

LBC : Light Blue Crest

VPP : Valeur Prédictive Positive

VPN : Valeur Prédictive Négative

Se : Sensibilité

Sp : Spécifité

OMS : Organisme Mondial de la Santé 


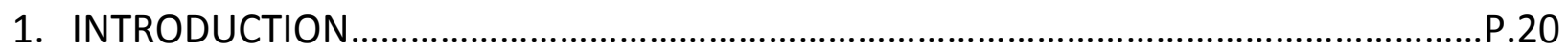

1.1. Anomalies de la muqueuse gastrique................................................................ 21

1.1.1. Muqueuse gastrique normale...................................................................P.21

1.1.2. Définition des lésions élémentaires..............................................................2.

1.1.2.1. Inflammation congestion / œdème..................................................P.22

1.1.2.2. Atrophie gastrique.........................................................................P. 23

1.1.2.3. Métaplasie intestinale gastrique........................................................ 23

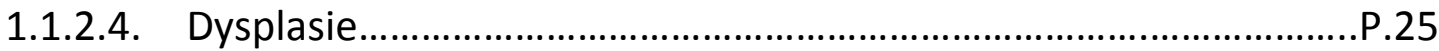

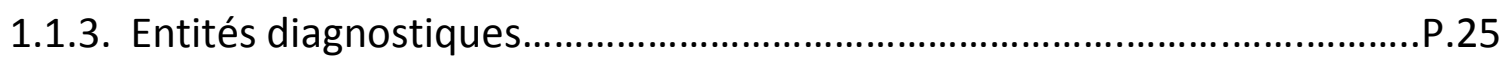

1.1.3.1. Gastrite aigue et chronique.........................................................P. 25

1.1.3.2. Gastropathie réactionnelle..............................................................26

1.1.3.3. Néoplasie........................................................................................ 27

1.1.4. Le compte rendu d'anatomopathologie pour les biopsies gastriques.....P.27

1.2. Muqueuse gastrique en chromo-endoscopie.....................................................P.28

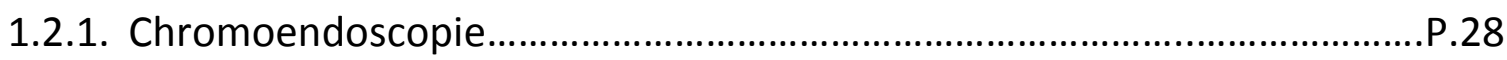

1.2.2. Muqueuse fundique normale et $\mathrm{NBI}$............................................................31

1.2.3. Muqueuse antrale normale et NBI.............................................................3.

1.2.4. Gastrite et atrophie en endoscopie...............................................................33

1.2.5. Métaplasie intestinale gastrique et $\mathrm{NBI}$........................................................34

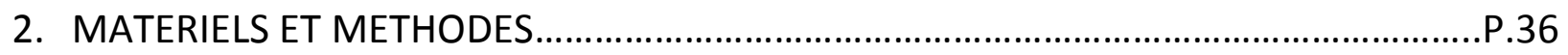

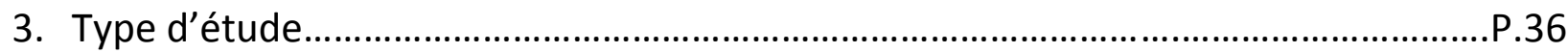

3.1. Critères d'inclusion et d'exclusion .........................................................................36

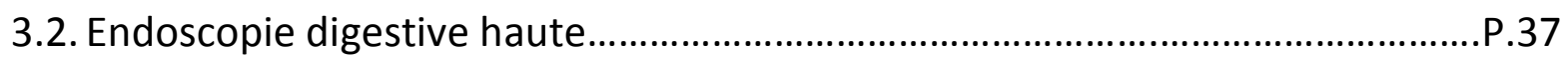

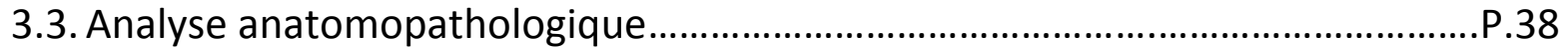

3.4. Analyse des vidéos d'endoscopie digestive haute................................................P. 38

3.4.1. $1^{\text {ère }}$ partie de lecture...................................................................................38

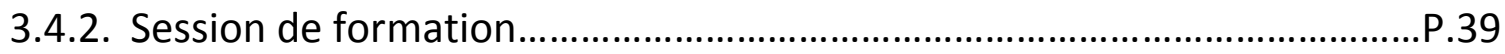

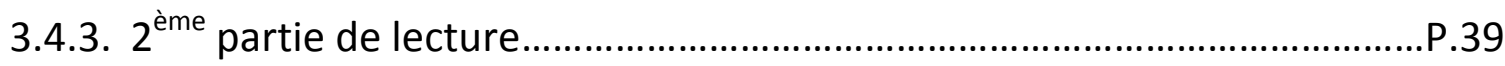

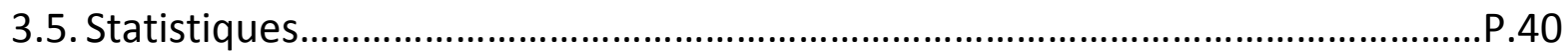


3.5.1. Détection de la métaplasie intestinale gastrique

3.5.2. Etude de l'évolution des résultats des lecteurs.......................................P.42

3.5.3. Etude des caractéristiques de la métaplasie intestinale gastrique...........P.42

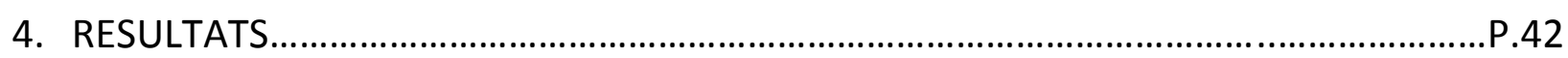

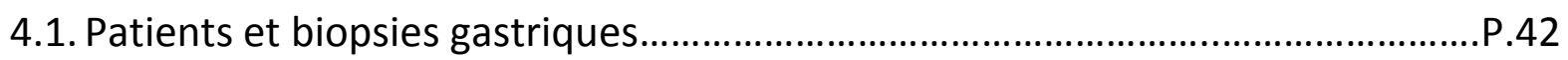

4.2. Vidéos sélectionnées pour les sessions.............................................................P.43

4.3. Caractéristiques de la métaplasie intestinale gastrique en NBI ........................P.44

4.4. Etude de l'évolution des résultats des lecteurs................................................P.45

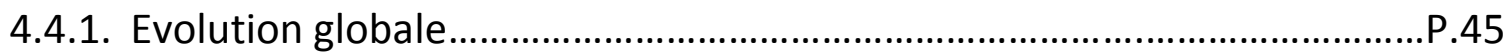

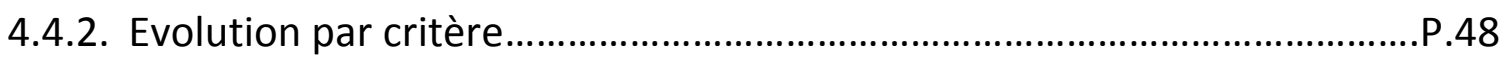

4.4.3. Evolution selon l'expérience des observateurs.......................................P.49

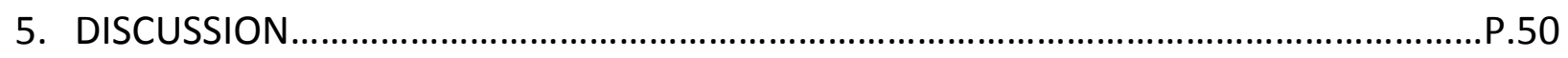

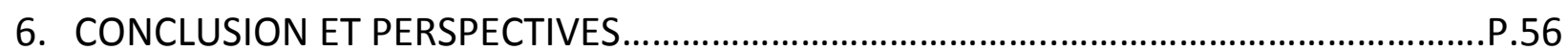

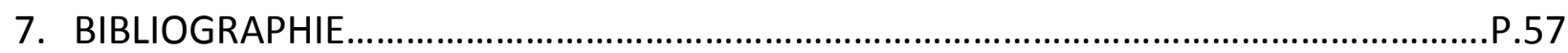

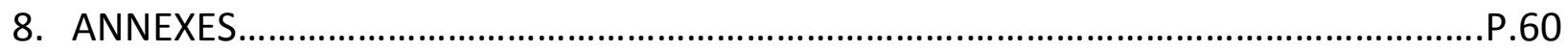




\section{INTRODUCTION}

Au niveau mondial, le cancer gastrique est le $4^{\text {ème }}$ cancer le plus fréquent chez I'homme, le $5^{\text {ème }}$ chez la femme, et la $3^{\text {ème }}$ cause de mortalité par cancer chez l'homme, la $5^{\text {ème }}$ chez la femme (1). En France, la diminution de l'incidence des cancers gastriques est possiblement parvenue à un plateau puisque le nombre de nouveaux cas était de 6550 en 2012 et de 6585 en 2015 (2). La moitié des cas est diagnostiquée après 75 ans (3) et le taux de survie à 5 ans est de l'ordre de $25 \%$, très lié au stade de la maladie. Cependant, malgré la diminution de l'incidence dans les pays les plus industrialisés, l'augmentation et le vieillissement de la population (4) en font un problème de santé publique.

Le cancer gastrique de type intestinal (d'après la classification de Lauren) est le type le plus fréquemment retrouvé, il est l'évolution terminale d'un processus allant de la gastrite chronique à l'atrophie gastrique et à la métaplasie intestinale. Il avait été suggéré qu'environ 50 \% des malades ayant une gastrite chronique évoluaient vers l'atrophie gastrique, que 8 \% évoluaient vers la présence de métaplasie intestinale compliquant l'atrophie et qu'enfin $1 \%$ évoluaient vers la dysplasie sévère et le cancer(5). D’après les recommandations européennes de 2012 (6), il est clairement établi que l'endoscopie en lumière blanche manque de fiabilité pour évaluer ou détecter les lésions précancéreuses de l'estomac. La chromo-endoscopie électronique telle que le Narrow-Band Imaging (NBI) est simple d'utilisation et a montré une bonne sensibilité et spécificité pour le diagnostic des lésions pré-néoplasiques, ainsi qu’une supériorité de détection de ces lésions par rapport à la lumière blanche (grade de recommandation B). De plus, du fait de la grande surface de la cavité gastrique, seulement de petites aires gastriques peuvent être biopsiées. La métaplasie intestinale pouvant avoir un caractère focal, les biopsies endoscopiques non ciblées peuvent être faussement rassurantes (7).

Actuellement, les performances diagnostiques de l'examen endoscopique restent en pratique limitées en France par défaut de connaissance de cette nouvelle sémiologie qui caractérise les lésions gastriques précancéreuses en NBI (6). 
Depuis l'étude de Uedo et al. (8), de nombreux travaux ont été menés pour étudier et définir une sémiologie précise permettant de détecter la métaplasie intestinale gastrique à l'aide du NBI.

L'objectif de cette étude était d'évaluer la performance d'un programme d'enseignement par internet pour la détection de la métaplasie intestinale gastrique dans les conditions d'endoscopie standard en France, chez des gastro-entérologues avec différents niveaux de formation.

\subsection{Anomalies de la muqueuse gastrique}

\subsubsection{Muqueuse gastrique normale}

L'épithélium de l'estomac est cylindrique unistratifié. II repose sur le chorion vasculaire de la lamina propria.

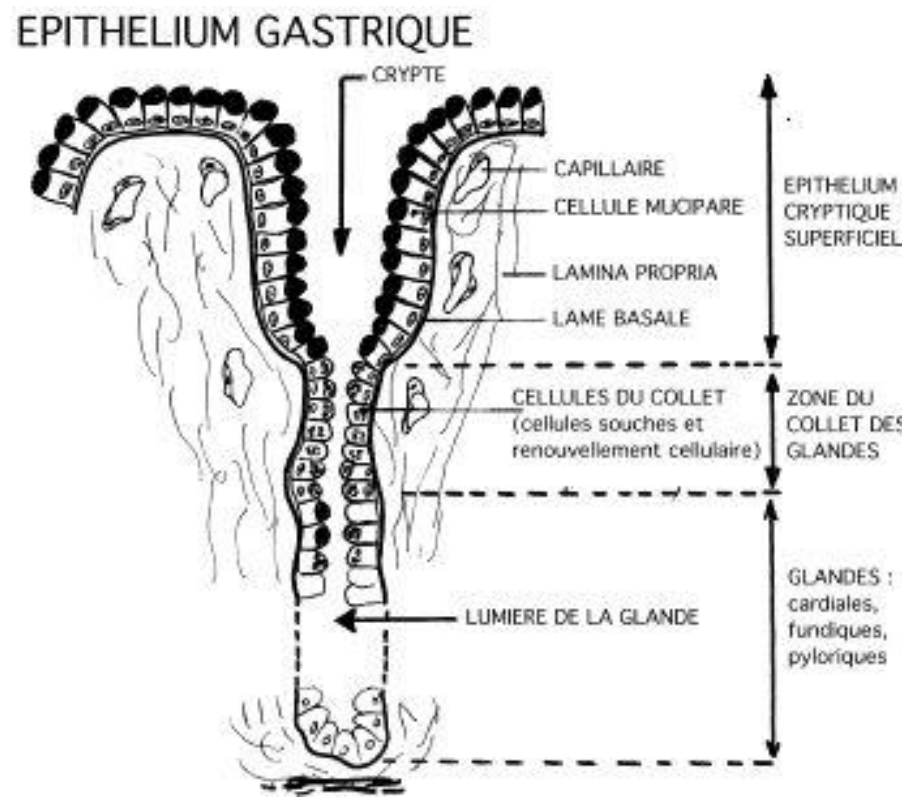

Figure 1 : schéma d'une glande gastrique

En surface, l'épithélium dessine des cryptes. A la base des cryptes, l'épithélium s'invagine profondément dans la lamina propria pour former des glandes (Figure 1) débouchant dans le fond des cryptes par une zone rétrécie, le collet, qui correspond à la zone de 
régénération et de prolifération mitotique à partir de laquelle s'effectue la totalité du renouvellement des cellules épithéliales.

La muqueuse gastrique présente 2 zones histologiques différentes (Figure 2) :

-la région fundique : les cryptes sont nombreuses, peu profondes et étroites. Le revêtement des glandes est constitué de cellules pariétales, plus nombreuses au niveau du collet et des cellules principales (cellules à pepsine) plutôt situé à la base des glandes.

-La région antro-pylorique : les cryptes sont hautes et étroites. Le revêtement des glandes est majoritairement constitué de cellules mucosécrétantes analogues aux cellules muqueuses du collet du corps et du fundus. On peut également retrouver quelques cellules pariétales, notamment à proximité du pylore.

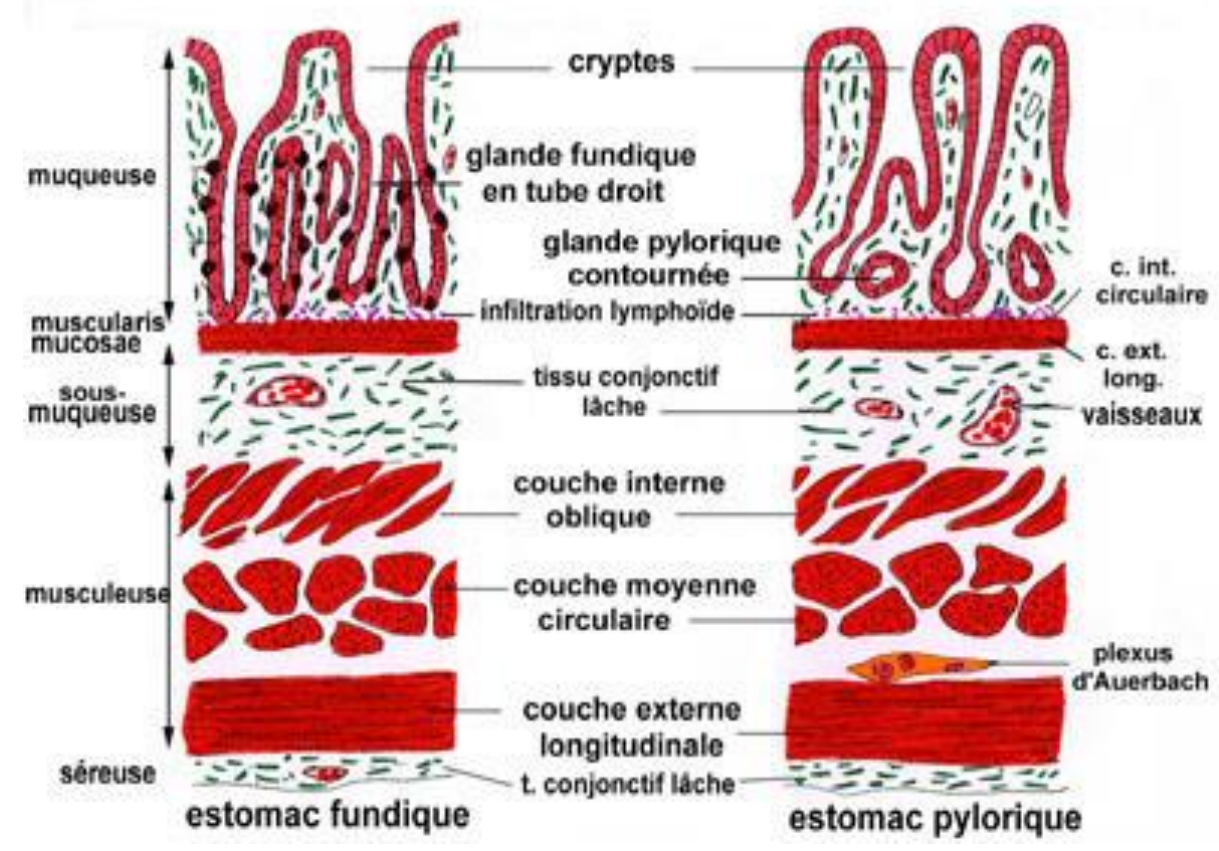

Figure 2 : Schéma représentant les 2 zones histologiques gastriques

\subsubsection{Définition des lésions élémentaires}

\subsubsection{Inflammation congestion / œdème}

On distingue plusieurs types d'infiltrat inflammatoire au sein de la muqueuse gastrique permettant notamment d’orienter le diagnostic étiologique de la gastrite. 
Les deux types d'infiltrations inflammatoires les plus fréquents sont :

- L'infiltrat inflammatoire lympho-plasmocytaire du chorion qui constitue un signe d'inflammation chronique.

- L'exocytose à polynucléaires neutrophile au sein du revêtement qui constitue un signe d'inflammation aiguë appelé également « activité ».

La congestion, représentée par une distension des vaisseaux, est souvent associée à de l'œdème. II s'agit de phénomènes peu spécifiques.

\subsubsection{Atrophie gastrique}

L'atrophie gastrique fait suite à l'évolution de la gastrite chronique, elle entraîne une disparition des unités glandulaires remplacées par une fibrose extensive de la lamina propria avec réduction de la masse glandulaire totale sans modification des types cellulaires originels. Elle peut aller de l'atrophie légère avec réduction de moins d'un tiers du volume des glandes à l'atrophie sévère avec réduction des deux tiers du volume des glandes.

La localisation de l'atrophie gastrique oriente son évolution ; si elle est située uniquement dans l'antre elle pourra évoluer en ulcère gastrique mais ne représente pas une lésion pré-néoplasique, alors que si elle se situe dans le corps gastrique ou si elle est pan-gastrique elle est reconnue comme lésion pré-cancéreuse de l’adénocarcinome gastrique de type intestinal (9).

Les 2 principales causes d'atrophie gastrique sont l'infection à Helicobacter Pylori (HP) (dans $85 \%$ des cas) et la gastrite auto-immune (de Biermer et se définit par une raréfaction des glandes gastriques.

\subsubsection{Métaplasie intestinale gastrique}

En anatomie pathologique, la métaplasie est la transformation d'un tissu cellulaire différencié en un autre tissu cellulaire différencié. II s'agit d'un phénomène adaptatif qui se produit le plus souvent en réponse à une agression tissulaire répétée et prolongée (inflammation, irritation mécanique ou chimique, infection), le tissu de remplacement étant mieux armé que le tissu original contre la dite agression. 
Dans la métaplasie, la structure et la fonction du nouveau tissu sont strictement normales, ce qui la distingue de la dysplasie (ou état précancéreux), bien qu'elle puisse évoluer secondairement vers ce stade si l'agression persiste.

La métaplasie ne peut être affirmée que par l'examen microscopique d'un prélèvement tissulaire.

La métaplasie intestinale gastrique est caractérisée par le remplacement de la muqueuse gastrique antrale et/ou fundique par une muqueuse de type intestinal, soit de type intestin grêle (métaplasie complète), soit de type côlon (métaplasie incomplète) (6). La métaplasie intestinale de type incomplet serait associée à un plus fort risque de cancer que la métaplasie de type complet. Cependant, en pratique on ne fait pas la distinction car il n'y a pas d'impact clinique formel.

Chronologiquement, cette lésion survient plus tardivement que l'atrophie gastrique, elle peut également survenir sur un terrain de gastropathie réactionnelle.

Histologiquement elle est caractérisée par la présence de cellules caliciformes, parfois associé à des cellules absorptives, des cellules de Paneth et/ou des cellules ciliées (figure 3).

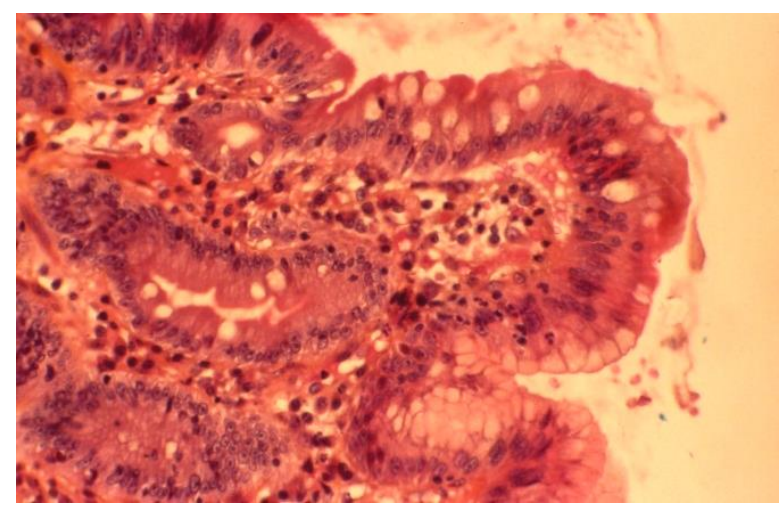

Figure 3 : coupe histologique d'une métaplasie intestinale gastrique

Comme l'atrophie, la métaplasie intestinale est d'autant plus associée au risque de cancer qu'elle est multifocale et non pas localisée à l'antre. En effet, il existe une grande variabilité d'évolution de cette lésion selon si elle se trouve uniquement dans l'antre, sans risque de dégénérescence, ou dans le corps gastrique seul ou associé à une 
localisation antrale, où elle majore le risque de développement de tumeur gastrique. Sa présence en bande le long de la petite courbure du cardia jusqu'au pylore est associée à un risque particulièrement élevé de cancer (10). Au stade de métaplasie intestinale, l'éradication d'HP ne permet pas la régression de la lésion. C'est une lésion irréversible.

\subsubsection{Dysplasie}

En anatomie pathologique on parle de dysplasie devant une altération acquise de l'architecture et de la fonction d'un tissu cellulaire à renouvellement rapide. La dysplasie possède une tendance globale à évoluer vers un cancer, ce qui en fait le synonyme d'état précancéreux.

Les anomalies cellulaires morphologiques qui la caractérisent concernent généralement des anomalies architecturales (retard de différenciation, désordre architectural), des anomalies cytologiques (mitoses, anomalies nucléaires), l'absence d'invasion (membrane basale intacte). En fonction de l'importance des anomalies morphologiques, on classe la dysplasie en dysplasie de bas grade et de haut grade (parfois trois catégories : légère/modérée/sévère). La dysplasie de haut grade est très proche du cancer. La dysplasie est toujours strictement localisée au tissu où elle naît et peut donc, si elle est dépistée à temps, bénéficier d'une prise en charge efficace.

\subsubsection{Entités diagnostiques}

\subsubsection{Gastrite aiguë et chronique}

On différencie la gastrite aiguë et la gastrite chronique.

-La gastrite aiguë est une inflammation aiguë de la muqueuse définie par des lésions anatomopathologiques. Elle peut être cliniquement latente ou bien se manifester par des épigastralgies. Les biopsies montrent des lésions souvent diffuses, multiples et de degré variable : œdème, hyperplasie fovéolaire (allongement des cryptes), hypercellularité de l'épithélium, exocytose à PNN pouvant aller jusqu'à l'ulcération, vasodilatation et congestion de la lamina propria. 
Les causes les plus fréquentes sont l'alcool, les médicaments (aspirine et AINS), la primo-infection à $\mathrm{H}$. pylori, l'ingestion de caustiques et les défaillances multiviscérales.

-La gastrite chronique signifie la présence à l'examen histologique de lésions inflammatoires et/ou atrophiques plus ou moins étendues et plus ou moins sévères. La gastrite chronique est susceptible d'aboutir progressivement à la disparition des glandes gastriques (atrophie gastrique). Les principales causes sont : l'infection à H. pylori, le reflux biliaire, la prise d'AINS ou d'aspirine au long cours et l'auto-immunité (maladie de Biermer).

La gastrite chronique à $\boldsymbol{H}$. Pylori pour sa part est très fréquente : 20 à $50 \%$ de la population adulte en France.

Après contamination oro-orale ou féco-orale le plus souvent au cours de l'enfance, l'infection se traduit d'abord par une gastrite aiguë qui évolue ensuite vers la chronicité dans la majorité des cas.

La gastrite chronique auto-immune est une pathologie rare, avec présence d'anticorps anti-cellules pariétales et anti-facteur intrinsèque.

Histologiquement, il y a destruction des glandes fundiques (gastrite atrophique fundique totale) avec souvent de la métaplasie antrale et de la métaplasie intestinale.

\subsubsection{Gastropathie réactionnelle}

Tableau commun à plusieurs entités : gastrite par reflux biliaire, gastrite post anastomotique, gastrite médicamenteuse (anti-inflammatoires non stéroïdiens), gastrite de l'alcoolisme chronique, gastrite post thérapeutique (radiothérapie ...), gastrite par compression extrinsèque, aspect cicatriciel après traitement d'une gastrite chronique.

Sur le plan histologique il existe un discret infiltrat inflammatoire lympho-plsamocytaire du chorion sans activité, une hyperplasie cryptique, une dystrophie épithéliale, un œdème, une congestion, et une ascension des fibres musculaires lisses pouvant être associée à de la fibrose. Il n'y a pas d'Helicobacter Pylori, pas de signe d'activité. 


\subsubsection{Néoplasie}

Il existe deux classifications pour définir les différents types d'adénocarcinomes gastriques : la classification de Lauren établie en 1965 qui définit trois types (intestinal, diffus et mixte) et la classification OMS, établie en 2000, qui est plus détaillée avec cinq principaux types : adénocarcinome tubuleux, papillaire, mucineux, carcinomes à cellules indépendantes (y compris le carcinome à cellules en bague à chaton et d'autres), carcinome mixte.

Les facteurs prédisposant sont environnementaux (consommation de tabac et alcool, facteurs socio-économiques), génétiques (polyposes), et les lésions prédisposantes (gastrite chronique atrophique, infection à Helicobacter Pylori et métaplasie, dysplasie, ulcères, polypes, moignons de gastrectomie).

\subsubsection{Interprétation des biopsies gastriques en anatomopathologie}

Le diagnostic de gastrite repose sur l'analyse anatomo-pathologique des biopsies gastriques.

Elles sont le plus souvent interprétées selon les critères de la classification de Sydney (Annexes 1 et 2), effectuée au Congrès mondial de Gastroentérologie de Sydney en 1990, et actualisée en 1996. Certains auteurs ont récemment proposé d'ajouter les stades OLGA et OLGIM (OLGA : Operative Link for Gastritis Assessment ; OLGIM : Operative Link on Intestinal Metaplasia).

D’autres part la classification de Sydney (et sa mise à jour de 1996) a également défini les recommandations suivantes :

- Nombre de biopsies à analyser : Deux biopsies antrales (à 2 ou $3 \mathrm{~cm}$ du pylore, au niveau de la petite et de la grande courbure distale), deux biopsies fundiques (au niveau du corps de l'estomac, à la partie moyenne de la grande courbure à $8 \mathrm{~cm}$ du cardia), et une biopsie angulaire. Ces biopsies devaient être communiquées dans trois pots différenciés.

Il est à noter qu'il existe une variabilité de ces recommandations, certains auteurs préconisent seulement 2 biopsies antrales et 2 biopsies fundiques (6). 
-Items histologiques à préciser : L'importance de l'infiltrat inflammatoire au sein du chorion, la présence de polynucléaires neutrophiles (traduisant l'activité de la gastrite), d'une atrophie muqueuse, de foyers de métaplasie intestinale, d'Helicobacter Pylori. -Description du stade évolutif de la gastrite : caractère aiguë ou chronique.

\section{Les Systèmes OLGA (Annexe 3) et OLGIM (Annexe 4)}

Ces systèmes d'évaluation de la muqueuse gastrique inflammatoire et atrophique ont été proposés par M. Rugge et un groupe de pathologistes experts. Ils permettent, par une approche systématique semi-quantitative et topographique, de typer la gastrite et de donner une indication du risque évolutif vers la dysplasie et le cancer. Il s'agit de systèmes potentiellement intéressants, mais semblant relativement complexes. Ils sont à l'heure actuelle relativement peu utilisés en routine diagnostique.

\subsection{Muqueuse gastrique et chromoendoscopie}

\subsubsection{Chromoendoscopie}

La chromoendoscopie est une technique de coloration de la muqueuse permettant d'affiner l'examen endoscopique en lumière blanche avec, selon les organes, un intérêt de détection, de caractérisation ou encore pour définir l'extension de la lésion. On oppose deux types principaux de colorations : les colorations par projection d'un colorant (de surface ou d'un colorant vital rentrant dans la cellule) à l'aide d'un cathéter spray; et les colorations virtuelles utilisant une lumière colorée ou un posttraitement de l'image. Les colorations réelles sont parfois complexes, et prennent du temps. A l'inverse, les colorations virtuelles comme le Narrow-Band Imaging (NBI) sont très simples d'utilisation, apportent des informations complémentaires sur le relief vasculaire mais ne peuvent pas remplacer les colorations réelles dans de nombreuses indications.

Les colorations virtuelles sont obtenues de 2 façons : 
- Soit en éclairant le tissu avec une certaine longueur d'onde, ce qui va révéler au sein de ce tissu des composants absorbant plus cette longueur d'onde (Narrow Band Imaging, NBI).

- Soit en travaillant le signal obtenu en lumière blanche classique (systèmes Fice, iScan).

Le système NBI, commercialisé par Olympus ${ }^{\circledR}$, est utilisé depuis 1999 et a été développé au Japon. Cette technique repose sur l'interposition entre la source lumineuse et le tissu éclairé d'un filtre permettant de sélectionner certaines bandes du spectre visible, choisies pour leurs propriétés physiques et optiques (11). Les systèmes NBI commercialisés actuellement utilisent une lumière constituée de deux bandes spectrales étroites, l'une dans le bleu centré à $415 \mathrm{~nm}$ et l'autre dans le vert centré à $540 \mathrm{~nm}$ (Figure 7).

L'image obtenue permet alors une analyse fine des structures superficielles et de la vascularisation muqueuse et sous muqueuse.
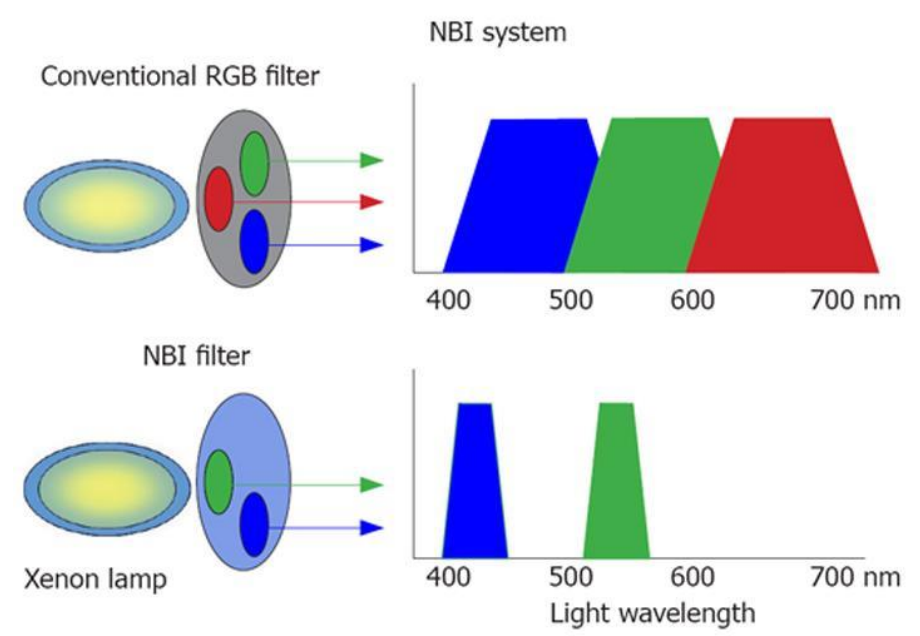

Figure 7 : Structure du système en lumière blanche et en NBI

Deux autres systèmes de coloration électronique sont commercialisés, il s'agit du Fuji Intelligent Color Enhancement (FICE, Fuji, Japon) et du système iScan (Pentax, Tokyo, Japon), fonctionnant comme le FICE. Le principe diffère dans l'éclairage du tissu par la lumière blanche. La sélection des bandes spectrales d'intérêt est réalisée à posteriori, 
de manière électronique. L'avantage de ce système est de choisir « à la carte » un réglage en fonction du tissu et de la pathologie étudiée. Le FICE et le iScan ont cependant moins été évalués.

L'utilisation de ces trois systèmes, NBI, FICE et iScan est particulièrement aisée et ne nécessite aucune préparation particulière. Le passage d'une image classique en lumière blanche à une image spécifique en bande spectrale étroite est instantané, obtenu par simple pression d'un bouton situé à la tête de l'endoscope. Ce processus est immédiatement réversible et peut être répété à loisir. La visualisation des reliefs muqueux et des structures peut être améliorée par l'utilisation simultanée d'un zoom optique, dit aussi magnification optique.

Les indications actuelles du NBI en France sont :

- Dépistage de zones suspectes de dysplasie de haut grade ou de cancer dans la surveillance d'endobrachyoesophages en associant coloration virtuelle par NBI et coloration réelle par acide acétique (avis d'experts).

- Dépistage des lésions pré-néoplasiques gastriques avec ou sans magnification (grade de recommandation $\mathrm{B}$ ).

- Caractérisation d'une lésion colique afin de prédire l'histologie de la lésion, son degré d'invasion et de décider de l'attitude thérapeutique adéquate.

Concernant la magnification en France, on retrouve deux types (12) :

- La magnification électronique ou digitale qui agrandit simplement l'image, avec comme conséquence diminution de la densité de pixels et une qualité d'image réduite. Avec leurs propres processeurs, les endoscopes conventionnels permettent une magnification digitale de $1,5 x$ à $2 x$.

- La magnification par zoom optique qui permet d'agrandir l'image tout en maintenant la qualité de l'image, le zoom maximal est de 150x. 
Les endoscopes les plus commercialisés en France sont ceux avec zoom digital pour des raisons financières et de rentabilité, l'examen détaillé par zoom optique est peu pratiqué en France.

\subsubsection{Muqueuse fundique normale et NBI}

En endoscopie avec magnification, la muqueuse gastrique fundique normale est caractérisée par un réseau de capillaires sous épithéliaux en forme de rayons de miel. Les points noirs au centre correspondent à l'ouverture des cryptes qui sont entourés par l'épithélium marginal de la crypte, qui est circulaire. On retrouve également la présence de veinules collectrices (figure 8).
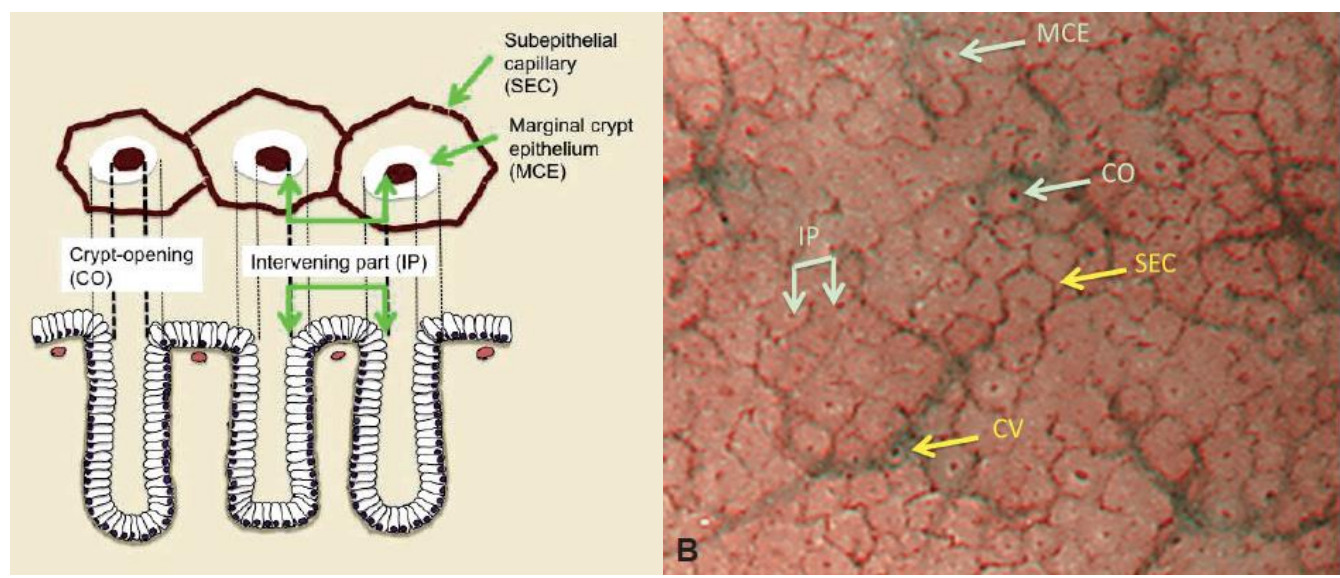

Figure 8 : muqueuse gastrique fundique normale a) schéma représentant la corrélation entre la microanatomie visualisée en NBI avec magnification (partie haute) et l'histologie (partie basse) dans la muqueuse fundique. b) muqueuse gastrique fundique vue une NBI avec magnification.

La muqueuse fundique en NBI et en l'absence de zoom apparait sous forme de points blancs sur fond brun, le fond brun représentant le réseau de capillaires sous épithéliaux en rayon de miel et les points blancs l'épithélium marginal des cryptes circulaire (figure 9). 


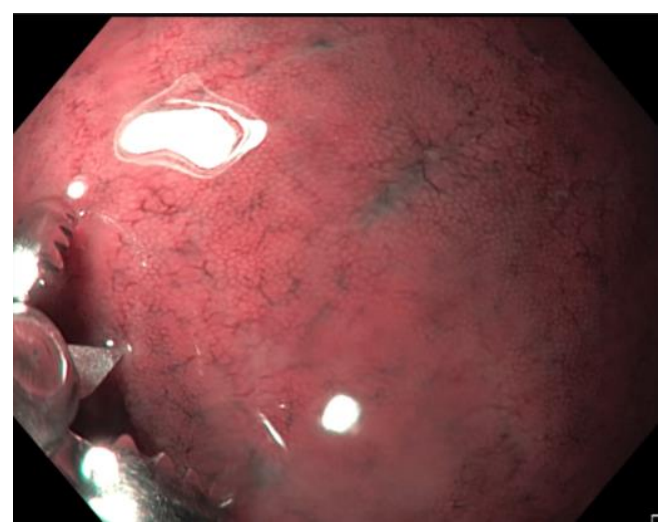

Figure 9 : muqueuse fundique normale vue en endoscopie NBI

\subsubsection{Muqueuse antrale normale et NBI}

En endoscopie avec magnification, la muqueuse gastrique antrale normale est caractérisée par un réseau de capillaires sous épithéliaux en forme de spires régulières, l'absence de veinules collectrices et un épithélium marginal des cryptes incurvé (figure 10).
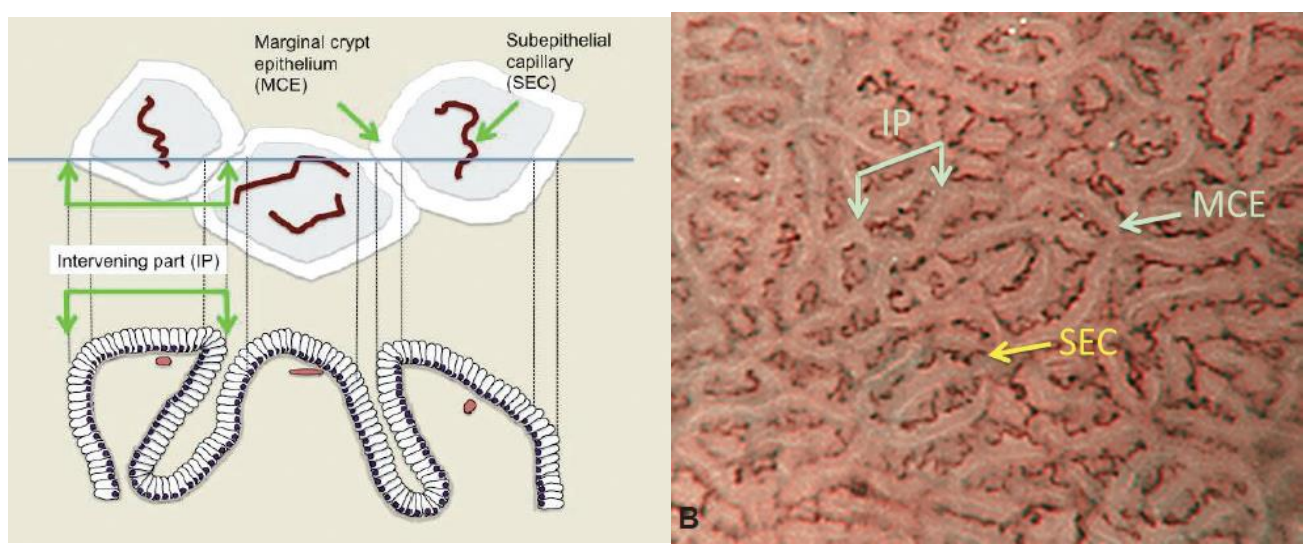

Figure 10 : muqueuse gastrique antrale normale a) schéma représentant la corrélation entre la microanatomie visualisée en NBI avec magnification (partie haute) et l'histologie (partie basse) dans la muqueuse antrale. b) muqueuse gastrique antrale vue une NBI avec magnification.

La muqueuse antrale en NBI et en l'absence de zoom apparait sous la forme d'un réseau blanc sur fond brun, le fond brun représentant les capillaires sous épithéliaux et le réseau blanc l'épithélium marginal des cryptes qui est incurvé (figure 11). 


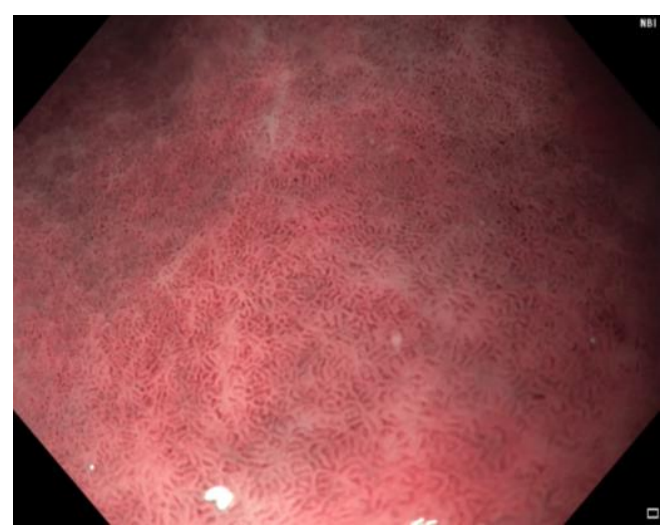

Figure 11 : Muqueuse antrale normale vue en endoscopie NBI

\subsubsection{Gastrite et atrophie gastrique en endoscopie}

En endoscopie en lumière blanche, les lésions élémentaires des gastrites se présentent sous sept aspects : hémorragique, catarrhal, pâleur de la muqueuse, érosions, surélévations, augmentation du calibre des plis, rigidités. Ces anomalies peuvent être localisées ou diffuses, isolées ou groupées. Elles ne préjugent pas de l'état histologique. L'interprétation en est difficile, et il faut tenir compte de l'âge, du contexte clinique, de l'évolution des lésions, des données biologiques et immunologiques.

Concernant la gastrite à Helicobacter Pylori, elle est caractérisée en NBI avec magnification par une perte des veinules collectrices, les cryptes élargies et entourées par de l'érythème (13) (Figure 12).

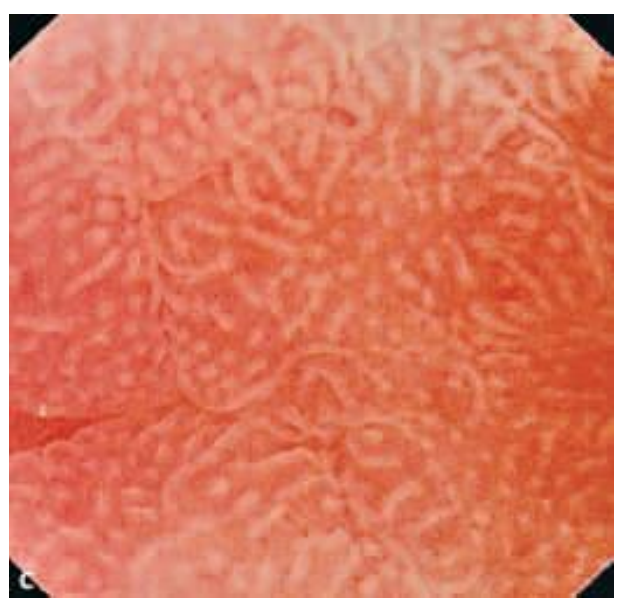

Figure 12 : Pattern d'une gastrite à Helicobacter Pylori en endoscopie lumière blanche 
L'atrophie gastrique est caractérisée en lumière blanche par un changement de couleur blanchâtre ou jaunâtre, des vaisseaux sous épithéliaux visibles, et l'absence de plis de la muqueuse (Figure 13).

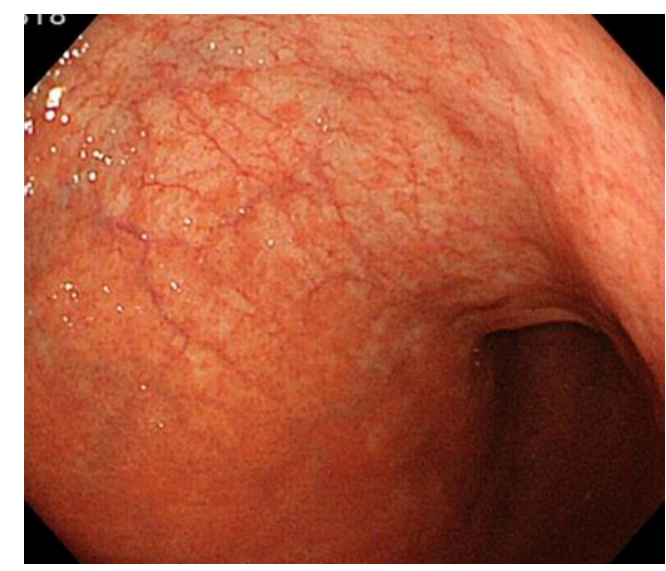

Figure 13 : Aspect d'atrophie gastrique en endoscopie en lumière blanche

\subsubsection{Métaplasie intestinale gastrique et NBI}

La métaplasie intestinale survenant dans les suites d'une gastropathie réactionnelle, d'une gastrite chronique à HP et d'une atrophie gastrique, tout diagnostic de ces entités devra faire rechercher de la métaplasie intestinale gastrique.

La métaplasie intestinale gastrique en endoscopie en lumière blanche peut apparaitre sous forme de lésions planes, plus blanches ; avec NBI elle peut se caractériser par trois entités qui peuvent être ou non associées :

- L'aspect tubulo-villeux des glandes : Pattern glandulaire tubulo-villeux bien tracé représenté par des combinaisons d'aires linéaires claires et foncées, et avec les aires plus claires ayant l'apparence d'être surélevées (14) (figure 12). 


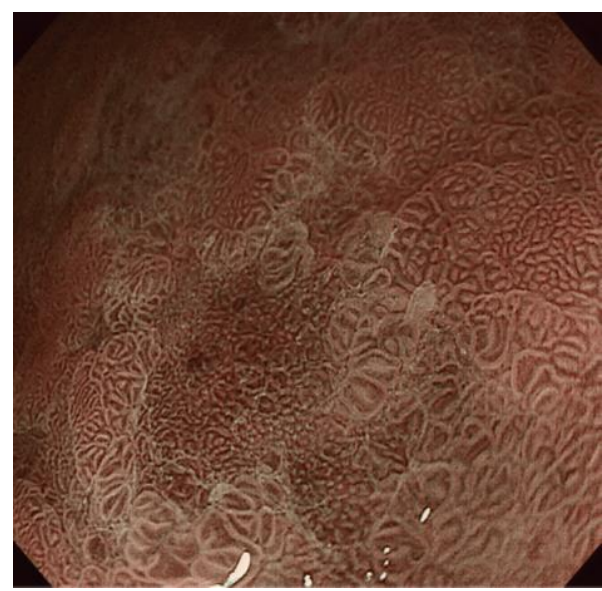

Figure 12 : aspect tubulo-villeux de la métaplasie intestinale gastrique en NBI

- La « white opaque substance » (WOS) : Aires de substance blanche rendant la vascularisation sous épithéliale opaque, visible en NBI. En anatomopathologie, cette substance blanche opaque correspond à des gouttelettes de lipides microscopiques (15) (figure 13).

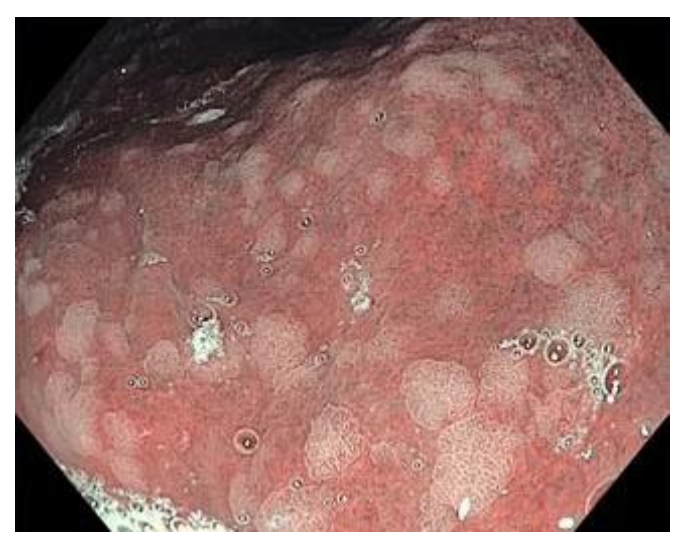

Figure 13 : aspect de white opaque substance de la métaplasie intestinale gastrique en NBI

- Les « light blue crest » (LBC) : Lignes bleu-blanchâtres au bord de l'épithélium des cryptes, sur des aires légèrement surélevées, visibles en NBI (figure 14). Les light blue crest ont initialement été décrites par Uedo et al. (8) en 2006, puis Pimentel-Nunes et al. qui ont montré les variations de cette caractéristique. 


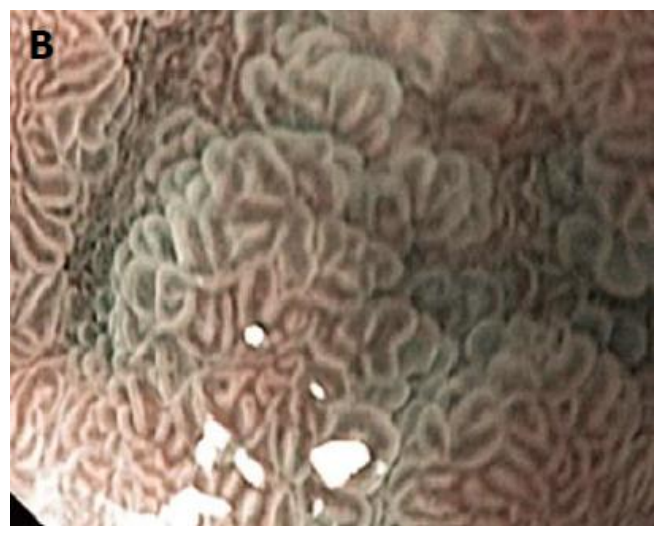

Figure 14 : aspect de light blue crest de la métaplasie intestinale gastrique en NBI

\section{MATERIELS ET METHODES}

\subsection{Type d'étude}

Il s'agit d'une étude prospective multicentrique, menée dans le département d’hépatoGastro-Entérologie du CHU de Nîmes, de janvier 2017 à juin 2018.

\subsection{Critères d'inclusion et d'exclusion}

Critères d'inclusion :

-Les vidéos du recueil initial étaient recueillies lors de gastroscopies de dépistage de métaplasie intestinale réalisée au CHU de Nîmes.

-Les observateurs des tests et de la formation power point étaient des gastroentérologues et internes en gastro entérologie pratiquant sur les sites de Nîmes (CHU) et Montpellier (CHU, CRLC ICM), avec des années d'expériences en endoscopie différentes ( $\leq 6$ ans, > 6ans d'expérience). 8 observateurs avaient une expérience $\leq 6 a n s$ en endoscopie et étaient inclus dans le groupe " juniors » : 5 internes et 3 CCA ; et 7 observateurs avaient une expérience $>6$ ans et étaient inclus dans le groupe « seniors » $: 6 \mathrm{PH}$ et un PU-PH.

Critères d'exclusion :

- vidéos du recueil initial de trop mauvaise qualité. 
- opérateurs ne répondant pas dans les délais impartis.

\subsection{Endoscopie digestive haute}

Les procédures d'endoscopie étaient menées par six endoscopistes sur le site de Nîmes, avec des vidéo-endoscopes Olympus GIF H180 et GIF H190, possédant un zoom digital de $1,5 x$ et avec une mise au point à $2 \mathrm{~mm}$. Aucun zoom n'était cependant utilisé pour la réalisation des vidéos.

Une fiche d'inclusion du patient était fournie, permettant de recenser les caractéristiques à l'entrée dans l'étude : Age, sexe, aspect macroscopique de la zone biopsiée, numéro de pot et localisation (antre/angulus/fundus) de la biopsie (annexe 5).

La gastroscopie se passait sous anesthésie générale. L'opérateur devait durant la période filmée :

- Filmer la lésion à biopsier en lumière blanche quelques secondes à distance puis à proximité pour qu'apparaissent le plus de détails possibles.

- Puis filmer la lésion à biopsier en NBI.

- Puis réaliser la biopsie.

Etaient biopsiées des lésions suspectes de métaplasie intestinales gastriques, des lésions autres et de la muqueuse normale.

Chaque film était analysé selon les trois critères endoscopiques en NBI de métaplasie intestinale gastrique : aspect tubulo-villeux des glandes, présence de « white opaque substance » et de " light blue crest ». Les vidéos également étaient classées selon une détection facile ou difficile de la métaplasie intestinale, en fonction du nombre de critères et de la facilité de détection des critères sur chaque vidéo.

Chaque film durait en moyenne 30 secondes. 


\subsection{Analyse anatomopathologique}

Chaque biopsie était ensuite envoyée au laboratoire d'anatomopathologie pour analyse. Sur chaque compte rendu était retenu : la présence ou l'absence de métaplasie intestinale gastrique, la présence d’autres lésions pouvant expliquer l'aspect macroscopique endoscopique.

Le diagnostic anatomopathologique était le critère de référence.

\subsection{Analyse des anomalies des vidéos d'endoscopie digestive haute}

Les vidéos de 30 biopsies gastriques sélectionnées selon la qualité de la vidéo et la présence ou absence de métaplasie ont été présentées lors de 3 sessions de 10 vidéos sous forme de power points à 15 endoscopistes de manière individuelle, afin de déterminer s'il y avait de la métaplasie intestinale gastrique sur chaque vidéo présentée. Avant de répartir les vidéos dans chaque session, elles étaient triées en vidéos de lecture facile et difficile. Pour chaque session, une répartition égale de la difficulté des vidéos et de la présence de métaplasie ou non était visée.

La lecture d'une session sans pause durait moins de 10 minutes. Les sessions et la formation power point étaient envoyées à l'ensemble des opérateurs par Google Drive.

\subsection{1. $1^{\text {ère }}$ partie de lecture}

Chaque lecteur avait un power point des vidéos de biopsies gastriques en lumière blanche et en NBI. Aucune information concernant la biopsie n'était fournie. Pour chaque vidéo, l'observateur devait noter s'il estimait qu'il y avait la présence de métaplasie ou non, et le degré de confiance pour sa réponse (faible ou élevé). Les réponses étaient notées sur la fiche de recueil (Annexe 6). 
Un pré test était réalisé : les 10 premières vidéos étaient d'abord lues, avant toute formation. Les réponses étaient renvoyées avant qu'ils ne reçoivent le power point de formation, et la correction détaillée étaient envoyés par la suite en même temps que le power point de formation.

\subsubsection{Session de formation}

A l'issue de la réception des réponses de la première session de vidéos, un cours sur power point avec support audio était envoyé. Celui-ci expliquait les différents critères endoscopiques permettant de détecter la métaplasie intestinale gastrique à l'aide du $\mathrm{NBI}$, des vidéos étaient inclues dans le power point en exemple pour chaque critère. Celles-ci étaient différentes des vidéos des trois sessions test.

Dans le même temps étaient envoyées les réponses corrigées de la session 1 , et la session 2 de vidéos tests à réaliser après lecture de la formation.

\subsubsection{Deuxième partie de la lecture}

Les 20 vidéos suivantes étaient lues par tranches de 10 (session 2 et 3). Les vidéos, sous forme de power point, étaient envoyées par mail, successivement, aux lecteurs. Comme pour la première session de vidéos, pour chaque vidéo, l'observateur devait noter s'il estimait qu’il y avait la présence de métaplasie ou non, et le degré de confiance pour sa réponse (faible ou élevé).

Les réponses de la session étaient envoyées par mail.

La correction de chaque session était un diaporama envoyé après réception des réponses, expliquant le diagnostic de chaque lésion et les critères présents permettant de détecter la métaplasie intestinale.

Le lecteur ne pouvait pas passer la session suivante sans avoir eu ses corrections (Figure 14). 


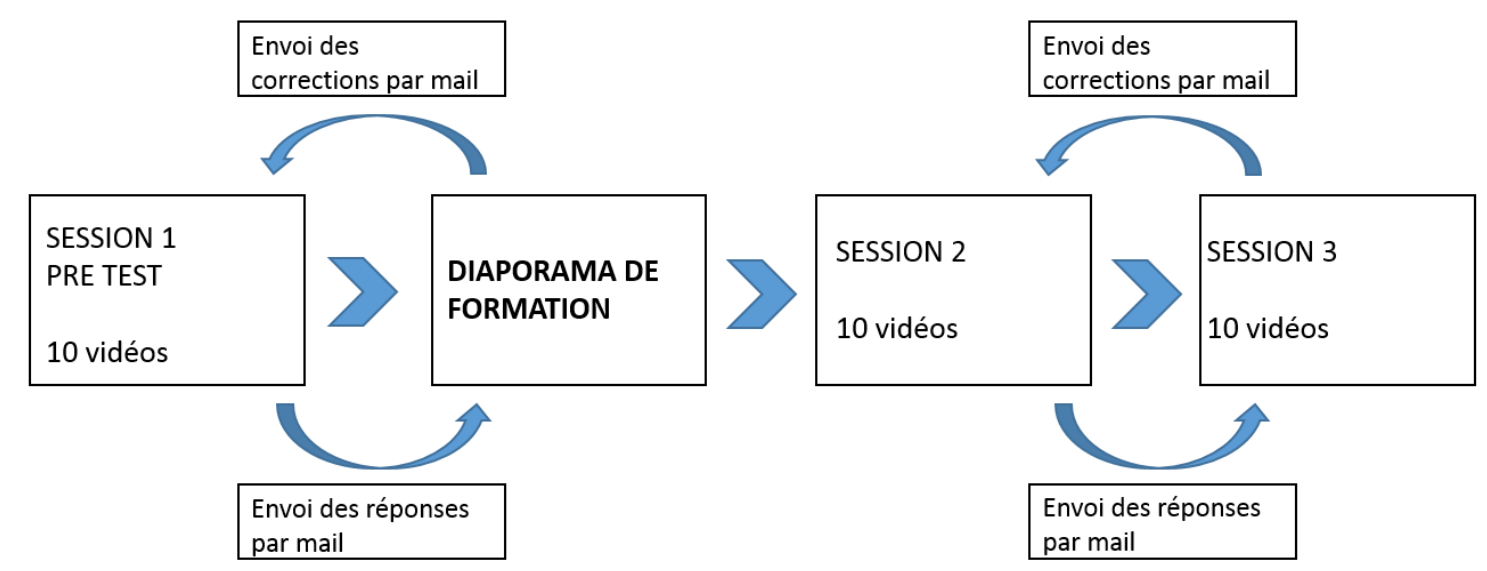

Figure 14 : Déroulement des lectures de diaporamas et corrections.

\subsection{Statistiques}

Cette étude était prospective.

Son objectif principal était : Evaluer la performance d'un programme d'enseignement par internet pour la détection de la métaplasie intestinale gastrique dans les conditions d'endoscopie standard en France, chez des gastro-entérologues avec différents niveaux de formation.

L'évaluation était réalisée selon les critères de jugement suivants :

- le score moyen de bonnes réponses, la sensibilité et la spécificité pour la détection de la métaplasie intestinale à chaque session.

- la différence de score, de sensibilité et de spécificité entre les sessions.

Ses objectifs secondaires étaient:

- Identifier les caractéristiques macroscopiques en NBI permettant de détecter la métaplasie intestinale gastrique.

-Evaluer les performances initiales d'un groupe de gastroentérologues pour la détection de la métaplasie intestinale gastrique par la sensibilité / spécificité / VPP (valeur prédictive positive) et VPN (valeur prédictive négative). 
-évaluer l'impact de la formation selon l'expérience en endoscopie des observateurs.

Les variables quantitatives étaient décrites par le nombre d'observations ( $N$ ), la médiane, le minimum, et le maximum, la moyenne et l'écart type.

Les tests T de Student ou Kruskal-Wallis étaient utilisés pour la comparaison des distributions des variables quantitatives selon le respect des conditions d'utilisation.

Les variables qualitatives étaient décrites par le nombre d'observations $(\mathrm{N})$ et la fréquence (\%) de chacune des modalités. Les catégories manquantes étaient dénombrées.

Le test du Chi-2 était utilisé pour la comparaison des proportions (ou test exact de Fisher si les fréquences attendues sont inférieures à 5).

Les intervalles de confiance à 95\% (IC95\%) étaient calculés par la méthode exacte Binomiale.

Tous les tests statistiques étaient bilatéraux et le seuil de significativité était fixé à $5 \%$ (ie. $p<0.05)$.

La sensibilité (Se) était la capacité à détecter la métaplasie intestinale gastrique lorsqu'elle était présente.

La spécificité (Sp) était la capacité à ne pas détecter la métaplasie chez les patients non atteints.

Les analyses statistiques ont été réalisées avec le soutien de l'Unité de Biométrie de I'Institut du Cancer de Montpellier avec le logiciel STATA v.13.

\subsubsection{Détection de la métaplasie intestinale gastrique}

Les réponses des lecteurs étaient comparées à l'analyse anatomopathologique, considérée comme valeur de référence. La valeur diagnostique des réponses était 
évaluée par le calcul du taux de bonnes réponses et le couple sensibilité/spécificité pour dépister la présence de métaplasie intestinale.

\subsubsection{Etude de l'évolution des résultats des lecteurs}

En l'absence d'étude similaire déjà menée, cette étude étant une étude de faisabilité, et le nombre d’opérateurs était déterminé selon la faisabilité de l'étude et 20 gastroentérologues de la région du Languedoc Roussillon ont été sollicités.

Des études de sous-groupes étaient réalisées selon le degré de confiance, l'expérience des lecteurs et les critères de détection de la métaplasie intestinale gastrique.

\subsubsection{Etude des caractéristiques de la métaplasie intestinale gastrique}

Une analyse descriptive des caractéristiques de la métaplasie intestinale gastrique sur les vidéos récoltées était réalisée. Les résultats statistiques étaient présentés sous forme de pourcentages et effectifs pour les 3 variables qualitatives.

\section{RESULTATS}

\subsection{Patients et biopsies gastriques}

Entre janvier 2017 à juin 2018, 126 biopsies gastriques ont été inclus dans l'étude.

Sur ces 126 biopsies filmées, 29 ont été retirées des analyses pour mauvaise qualité de la vidéo ou non-respect du protocole pour réaliser le film.

97 films de 50 patients ont donc servi de base de données pour réaliser les trois sessions de test, et déterminer la sensibilité, spécificité, valeur prédictive positive et valeur prédictive négative des trois critères.

Sur 42 des 97 films était présente de la métaplasie intestinale gastrique à l'anatomopathologie. Les biopsies gastriques étaient donc analysables pour définir la 
spécificité et sensibilité des critères permettant de retrouver la métaplasie intestinale gastrique en NBI. Parmi ces 42 films, 26 étaient classés avec de la métaplasie intestinale gastrique à l'aspect macroscopique durant l'endoscopie.

Les 55 autres films ne retrouvaient pas de métaplasie intestinale gastrique. Parmi ces 55 films, 8 étaient classés avec de la métaplasie intestinale gastrique à l'aspect macroscopique durant l'endoscopie. (Figure 15)

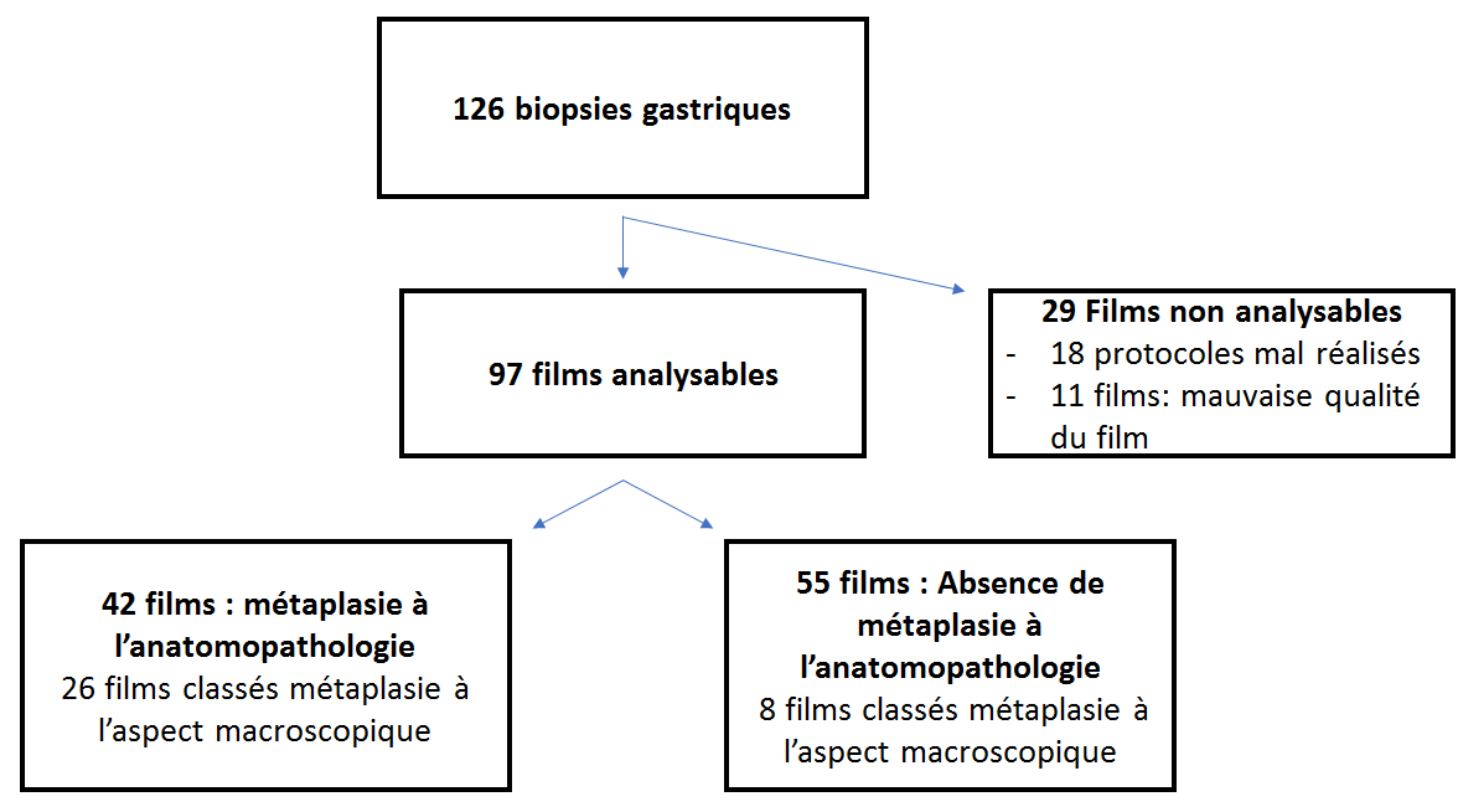

Figure 15 : flow chart

Nous avons également regardé sur quel terrain anatomopathologique survenait la métaplasie intestinale. Sur les 42 biopsies de métaplasie, 39,5\% retrouvait des lésions de gastrite chronique (17/43), 41,8\% retrouvait des lésions de gastropathie réactionnelle (18/43), 11,6\% de dysplasie de bas et haut grade (5/43), 4,6\% de muqueuse remaniée aspécifique (2/43).

\subsection{Vidéos sélectionnées pour les sessions}

Le même nombre de vidéos estimées « faciles » ou « difficiles » pour la détection de la métaplasie intestinale était présent dans chaque session. Les vidéos sélectionnées 
étaient de bonne et moyenne qualité. II y avait $50 \%$ des vidéos qui présentaient de la métaplasie dans les sessions 1 et 2, et 70\% dans la session 3. Parmi les 17 vidéos présentant de la métaplasie, 7 se situaient dans le fundus (41,2\%), 9 dans l'antre (53\%), 1 dans l'angle (5,9\%).

\subsection{Caractéristiques de la métaplasie intestinale gastrique en NBI}

La sensibilité, spécificité, valeur prédictive positive et valeur prédictive négative ont été calculées pour chaque critère avec leurs intervalles de confiance à 95\% à partir des 97 films de biopsies gastriques analysables (Tableau 1).

-Pour le critère « aspect tubulo-villeux des glandes ", la sensibilité était de 76\%, la spécificité de $83 \%$, VPP 78\%, VPN 82\%.

-Pour le critère « white opaque substance », la sensibilité était de 19\%, la spécificité $100 \%$, VPP $100 \%$, VPN $62 \%$.

-Pour le critère « light blue crest », la sensibilité était de 21\%, la spécificité 94\%, VPP 75\%, VPN $61 \%$

\begin{tabular}{lllll}
\hline Critère & sensibilité (\%) & spécificité (\%) & VPP (\%) & VPN (\%) \\
\hline Tubulovilleux & $76(60,5-87,9)$ & $83(71,1-92,2)$ & $78(62-89)$ & $82(70-91)$ \\
WOS & $19(9-34)$ & 100 & 100 & $62(51-72)$ \\
LBC & $21(10-37)$ & $94(85-99)$ & $75(43-95)$ & $61(50-72)$ \\
\hline VPP=valeur prédictive positive ; VPN= valeur prédictive négative, WOS=white opaque substance ; LBC= light blue \\
crest
\end{tabular}

Tableau 1 : Validité des critères et intervalles de confiance à 95\% pour la détection de la métaplasie intestinale gastrique pour tous les films analysables. 


\subsection{Etude de l'évolution des résultats des lecteurs}

\subsubsection{Evolution globale}

Les tableaux 2 et 3 représentent les résultats concernant l'évolution globale des marqueurs de validité (sensibilité et spécificité), la moyenne et la médiane au cours des 3 sessions, associées à la significativité statistique de leur évolution.

SESSION 1

SESSION 2

SESSION 3

\begin{tabular}{l|lll}
\hline SENSIBILITE & 0.68 & 0.75 & 0.75 \\
SPECIFICITE & 0.72 & 0.85 & 0.84 \\
MOYENNE & $7(1,92)$ & $8(1,19)$ & $7,8(1,14)$ \\
MEDIANE & $7(4-10)$ & $8(5-9)$ & $8(6-9)$
\end{tabular}

Tableau 2 : Sensibilité, Spécificité et taux de bonnes réponses exprimées en moyennes (dérivation standard) et médiane (rang minimal et maximal) pour chaque session

\begin{tabular}{|l|l|l|l|}
\hline Significativité $\boldsymbol{p}$ & Différence S1-S2 & Différence S2-S3 & Différence S1-S3 \\
\hline sensibilité & 0.317 & 0.317 & 0.317 \\
\hline Spécificité & 0.317 & 0.317 & 0.317 \\
\hline moyenne & 0.035 & 0.969 & 0.099 \\
\hline
\end{tabular}

Tableau 3 : significativité statistique d'évolution entre chaque session concernant la sensibilité, la spécificité et la moyenne.

Le graphe 1 présente l'évolution des résultats des 15 lecteurs au cours des 3 sessions de lectures des vidéos de biopsies gastriques : 


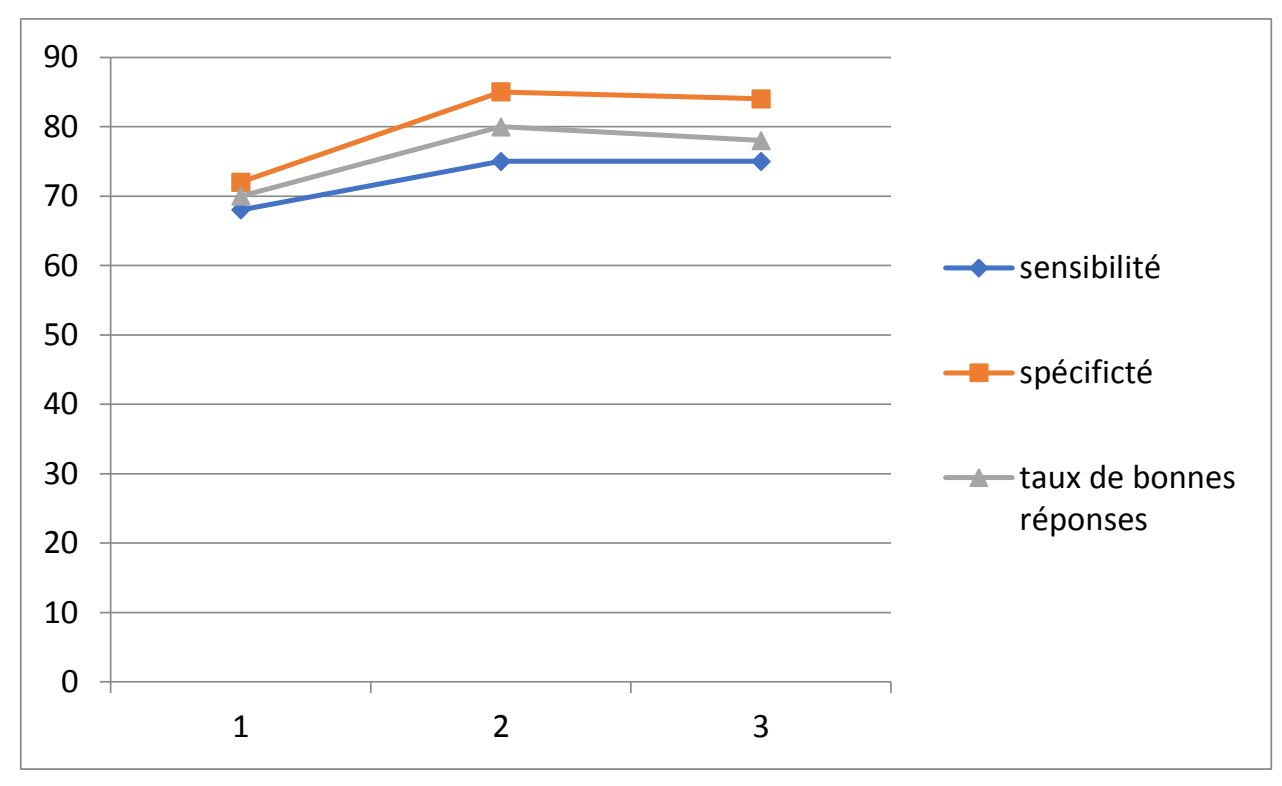

Graphe 1: Evolution de la sensibilité et la spécificité pour le diagnostic de métaplasie intestinale gastrique, et le taux de bonnes réponses

Le taux de bonnes réponses augmentait de façon significative de $70 \%$ à $80 \%$ entre la session 1 et $2(p=0.035) .9$ opérateurs sur 15 s'amélioraient, dont 6 avaient une expérience en endoscopie $\leq 6 a n s$, et 3 opérateurs avaient plus de 6 ans d'expérience.

Le taux de bonne réponse par vidéo était calculé (tableau 4).

Nous avons considéré qu'un taux de bonnes réponses élevées pour une vidéo correspondait à un taux $>85 \%$. Il existait trois vidéos avec un taux de bonnes réponses élevé dans la session 1, cinq vidéos dans la session 2, six vidéos dans la session 3.

Par ailleurs il existait trois vidéos qui avaient moins de $50 \%$ de bonnes réponses. Une vidéo se situait dans la session 2, et deux vidéos dans la session 3 . 


\begin{tabular}{|c|c|c|c|c|c|c|c|c|c|c|c|c|c|}
\hline & \multicolumn{4}{|c|}{ Session 1 } & \multicolumn{3}{c|}{ Session 2 } & \multicolumn{3}{c|}{ Session 3} \\
\hline $\begin{array}{c}\text { Taux } \\
\text { bonnes } \\
\text { réponses }\end{array}$ & TBV & LBC & WOS & $\begin{array}{c}\text { Taux } \\
\text { bonnes } \\
\text { réponses }\end{array}$ & TBV & LBC & WOS & $\begin{array}{c}\text { Taux } \\
\text { bonnes } \\
\text { réponses }\end{array}$ & TBV & LBC & WOS \\
\hline $\begin{array}{c}\text { Vidéo } \\
1\end{array}$ & 67 & Oui & Oui & Non & 80 & Oui & Non & Non & 47 & Oui & Non & Non \\
\hline $\begin{array}{c}\text { Vidéo } \\
2\end{array}$ & 60 & Non & Non & Non & 93 & Oui & Non & Oui & 93 & Oui & Non & Oui \\
\hline $\begin{array}{c}\text { Vidéo } \\
3\end{array}$ & 87 & Non & Non & Non & 93 & Non & Non & Non & 60 & Oui & Non & Non \\
\hline $\begin{array}{c}\text { Vidéo } \\
4\end{array}$ & 87 & Oui & Non & Oui & 67 & Oui & Non & Non & 100 & Oui & Non & Oui \\
\hline $\begin{array}{c}\text { Vidéo } \\
5\end{array}$ & 53 & Oui & Non & Non & 93 & Non & Non & Non & 93 & Non & Non & Non \\
\hline $\begin{array}{c}\text { Vidéo } \\
6\end{array}$ & 53 & Oui & Non & Non & 47 & Oui & Oui & Non & 87 & Oui & Non & Non \\
\hline $\begin{array}{c}\text { Vidéo } \\
7\end{array}$ & 93 & Non & Non & Non & 93 & Non & Non & Non & 47 & Oui & Non & Non \\
\hline $\begin{array}{c}\text { Vidéo } \\
8\end{array}$ & 53 & Non & Non & Non & 67 & Non & Non & Non & 93 & Non & Non & Non \\
\hline $\begin{array}{c}\text { Vidéo } \\
9\end{array}$ & 6 & Non & Non & Non & 87 & Oui & Oui & Non & 67 & Non & Non & Non \\
\hline $\begin{array}{c}\text { Vidéo } \\
10\end{array}$ & 80 & Oui & Oui & Non & 80 & Non & Non & Non & 93 & Oui & Non & Non \\
\hline
\end{tabular}

Tableau 4 : taux de bonnes réponses par vidéo exprimé en pourcentage (\%), et présence des critères TBV (tubulovilleux), LBC (light blue crest), et WOS (White opaque substance) à chaque session.

La sensibilité globale pour le diagnostic de la métaplasie intestinale gastrique passait de $68 \%$ à $75 \%$, et la spécificité de $75 \%$ à $84 \%$ entre la session 1 et 3 , ces augmentations n'étaient pas significatives.

Lorsque l'indice de confiance était élevé, la sensibilité augmentait de $83 \%$ à $88 \%$ à la $2^{\text {ème }}$ session mais n'augmentait plus ensuite. Cette augmentation n'était pas significative $(p=0,29)$. Le taux de bonnes réponses progressait peu (de 78 à $82 \%)$. 


\subsubsection{Evolution par critère}

Le Graphe 2 représente l'évolution des taux de bonnes réponses par critère permettant de détecter la métaplasie intestinale gastrique.

La sensibilité et la spécificité par critère n'ont pas pu être établies devant l'absence de certains critères lorsqu'il n'y avait pas de métaplasie présente sur les vidéos.

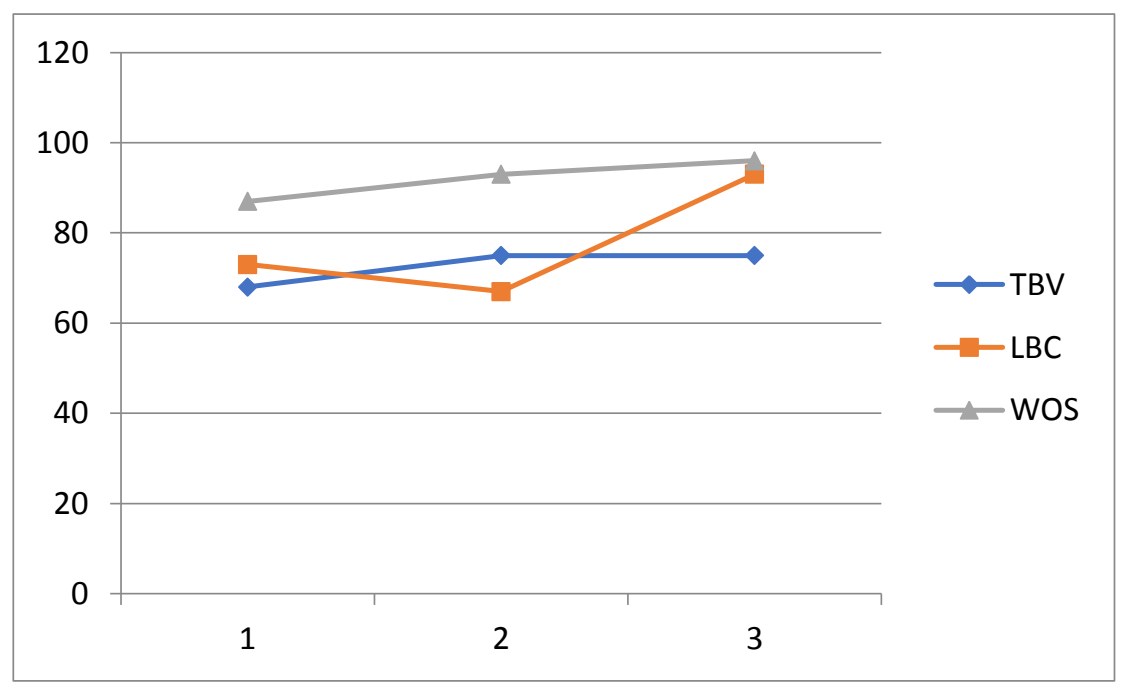

Graphe 2 : Evolution du taux de bonnes réponses selon le critère étudié. TBV=tubulovilleux, $L B C=$ light blue crest, WOS=white opaque substance

Lorsque le critère « tubulo-villeux » était présent pour la détection de la métaplasie intestinale gastrique, la sensibilité augmentait de $68 \%$ à $82 \%$ entre la session 1 et 3, et le taux de bonnes réponses passait de $68 \%$ à $75 \%$ à la session 2 et 3 . Aucune augmentation n'était statistiquement significative.

Lorsque le critère « light blue crest » était présent pour la détection de la métaplasie intestinale gastrique, le taux de bonnes réponses augmentait de $73 \%$ à $93 \%$ entre la session 1 et 3 , cette augmentation était statistiquement significative $(p=0,013)$. La correction de la session 2 permettait également un apprentissage puisque la détection était améliorée de façon significative entre la session 2 et $3(p=0,006)$. 
Lorsque le critère « white opaque substance » était présent pour la détection de la métaplasie intestinale gastrique, le taux de bonnes réponses augmentait de $9 \%$ pour atteindre $96 \%$ à la session 3.

\subsubsection{Evolution selon l'expérience des observateurs}

Le graphe 3 présente l'évolution des résultats des 8 lecteurs ayant une expérience en endoscopie $\leq 6$ ans au cours des 3 sessions de lectures des vidéos de biopsies gastriques.

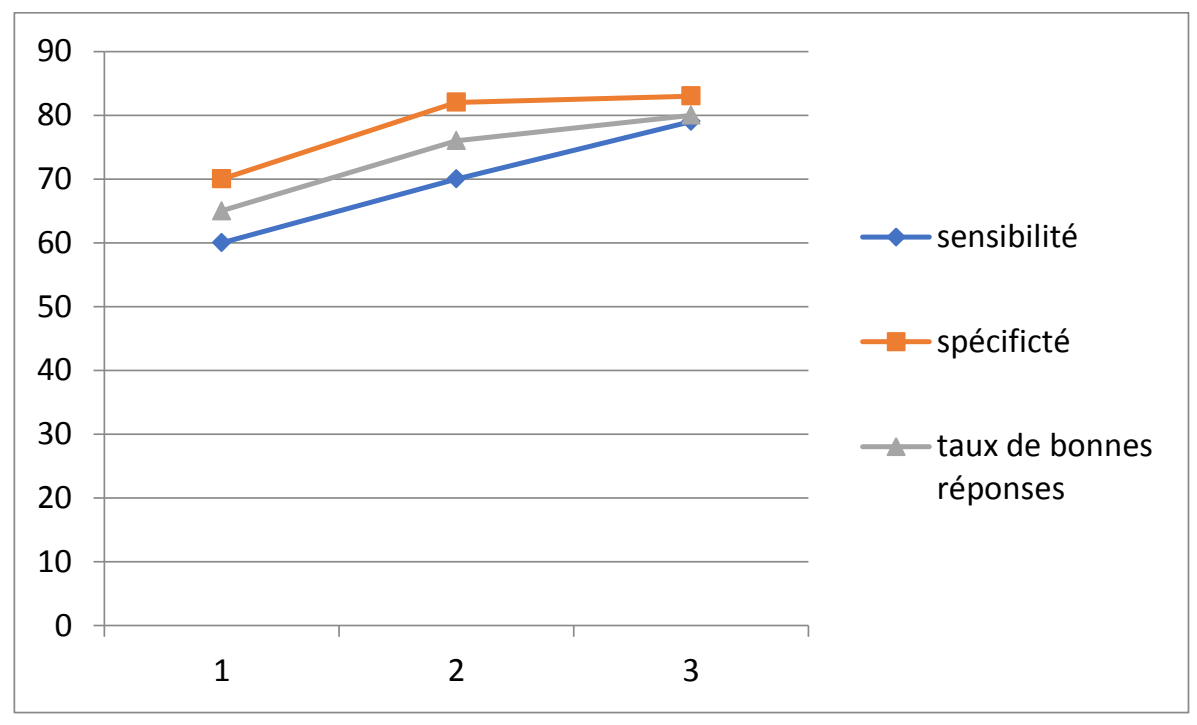

Graphe 3 : Evolution de la sensibilité et la spécificité pour le diagnostic de métaplasie intestinale gastrique, et le taux de bonnes réponses dans le groupe <6ans d'expérience en endoscopie

La sensibilité passait de $60 \%$ à $70 \%$, la spécificité de $70 \%$ à $83 \%$ et le taux de bonnes réponses de $65 \%$ à $80 \%$. Ces augmentations n'étaient pas statistiquement significatives.

Le graphe 4 présente l'évolution des résultats des 8 lecteurs ayant une expérience en endoscopie > 6 ans au cours des 3 sessions de lectures des vidéos de biopsies gastriques. 


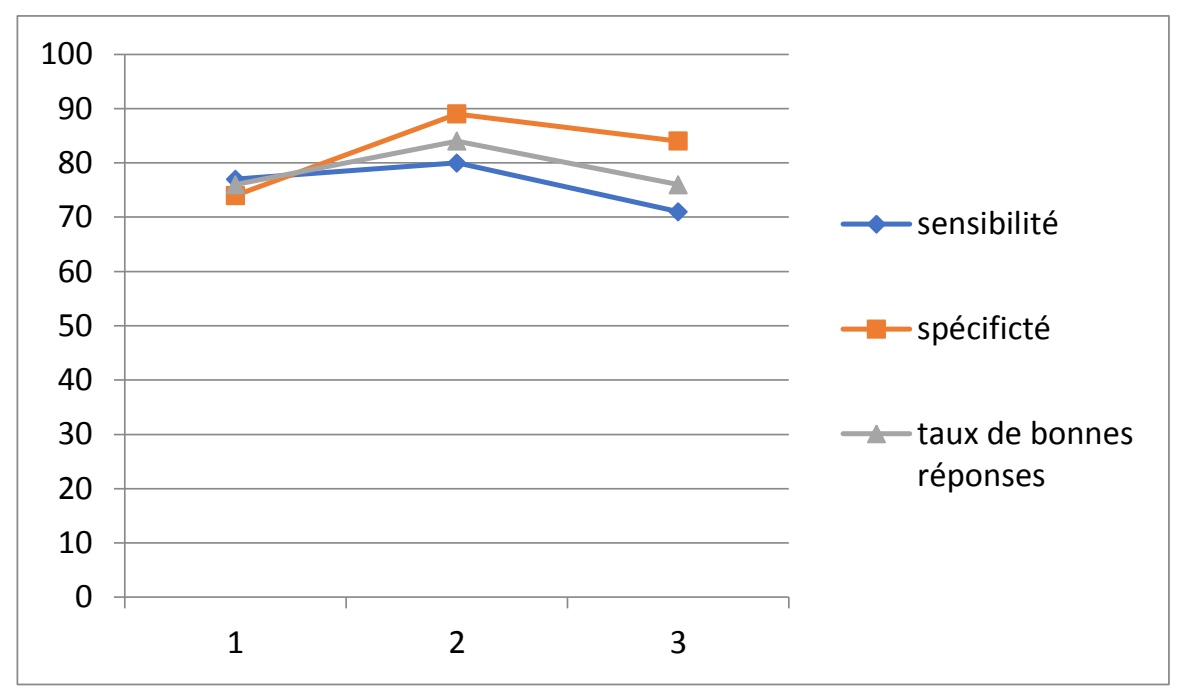

Graphe 4: Evolution de la sensibilité et la spécificité pour le diagnostic de métaplasie intestinale gastrique, et le taux de bonnes réponses dans le groupe >6ans d'expérience en endoscopie

Lorsque les opérateurs avaient plus de 6 ans d'expérience en endoscopie, il existait une augmentation non significative de la sensibilité, spécificité et du taux de bonnes réponses entre la session 1 et 2, qui ne persistait pas lors de la session 3 pour revenir aux taux de base ; sauf pour la spécificité qui augmentait de 12\% (respectivement $71 \%$, $86 \%$ et $76 \%)$.

En comparant le taux de bonnes réponses du groupe junior et du groupe sénior, il n'existait pas de différence significative à aucune des 3 sessions.

\section{DISCUSSION}

Le cancer gastrique reste un problème de santé publique par sa fréquence et sa mortalité avec un pronostic sombre à 5 ans. La relation entre métaplasie intestinale gastrique et adénocarcinome gastrique est bien établie depuis plusieurs années, et le risque de développer un adénocarcinome gastrique lorsque cette lésion est retrouvée peut être multiplié par 10 (16). De multiples études japonaises et une étude occidentale retrouvent des résultats similaires avec $8,4 \%$ des malades ayant une métaplasie intestinale qui développent un cancer gastrique dans les 10 ans (17). 
Au Japon, l’incidence élevée du cancer gastrique (dix fois supérieure à celle observée en France) a conduit à mettre en place dans ce pays une stratégie de dépistage endoscopique des cancers gastriques en population générale. Par cette stratégie de dépistage systématique, un pourcentage élevé de diagnostic de cancers gastriques superficiels au Japon (plus de $50 \%$ des cas de cancers gastriques diagnostiqués) était identifié. En comparaison avec la France, où le dépistage en population générale du cancer gastrique n'est pas pratiqué en raison d'une plus faible incidence de cancer, le pourcentage des formes superficielles identifiées par endoscopie est compris entre 5 et $10 \%(2)$.

Il est donc nécessaire de se former à détecter cette lésion le plus précocement possible, notamment par l'apport de nouvelles techniques endoscopiques telle que le NBI.

Notre étude était une étude de faisabilité, qui regroupait plusieurs avantages. D'une part, il s'agissait de la première étude française sur ce sujet, qui proposait un format avec un pré-test, une formation et un post-test. D'autre part, elle se rapprochait de très près des conditions réelles de réalisation des endoscopies digestives hautes en France, par le fait que les images proposées étaient des vidéos, qu'aucun zoom n'était employé tout comme dans la plupart des pôles d'endoscopie en France, et qu'aucun agent antispasmodique ni mucolytique n'était employé lors de la réalisation des endoscopies. De plus, elle proposait une formation informatique transmise par internet permettant de diffuser les connaissances de manière innovante, et qui s'avère devenir un excellent outil pour améliorer le rendement du diagnostic (18). Enfin, nous retrouvions déjà une augmentation significative du taux de bonnes réponses après la formation avec seulement 15 opérateurs.

Trois critères étaient choisis pour caractériser la métaplasie intestinale gastrique, basés sur une analyse de la littérature dédiée à ce sujet :

-le critère « tubulo-villeux », décrit initialement dans l'étude de Bansal et al. (14), s'associait à une bonne sensibilité (80\%), spécificité (100\%) et valeur prédictive positive (100\%). Ces données étaient confirmées par l'étude portugaise de Pimentel-Nunes et al. 
(19). Dans notre étude, ce critère retrouvait une bonne validité (sensibilité 76\%, spécificité 83\%), ce qui confortait les données déjà rapportées.

-le critère « light blue crest » décrit initialement dans l'étude de Uedo et al. (8) qui retrouvait une sensibilité de $89 \%$, une spécificité de $93 \%$ et une valeur prédictive positive de $91 \%$. La validité de ce critère a ensuite été étudié dans de nombreux travaux (19-22). Dans notre étude, la sensibilité était faible à 21\%, pour une spécificité haute à 94\%. Ces résultats peuvent être expliqués par plusieurs éléments : Tout d’abord le zoom optique utilisé dans la plupart des études était au minimum x80 et allait jusque x115. Les endoscopes utilisés dans notre étude correspondaient à la majorité des endoscopes utilisés en France avec un zoom digital faible de 1,5x. Une seule autre étude, portugaise, utilisait également des endoscopes avec zoom optique faible x1,5 pour détecter les light blue crest et obtenait une sensibilité de $48 \%$ et une spécificité de $96 \%$ (19).

De plus, la plupart des études utilisaient également des agents antispasmodiques et mucolytiques afin de limiter les mouvements d'estomac et les sécrétions gastriques qui peuvent gêner à la détection des LBC. Ces agents ne sont pas utilisés en pratique courante lors des endoscopies digestives hautes, et donc n'étaient pas utilisés dans notre étude car nous souhaitions réaliser une étude se rapprochant le plus possible des conditions réelles.

Enfin, la plupart des études étaient réalisées sur des photos des zones biopsiées. Ce qui rendait la détection du signe plus facile que sur une vidéo où les mouvements de contraction de l'estomac peuvent perturber la visualisation des LBC.

- Le critère « white opaque substance » fut décrit initialement pour distinguer les adénomes des carcinomes (23), puis étudié pour la détection de la métaplasie intestinale. Dans une étude japonaise, la sensibilité et spécificité de la WOS pour détecter la métaplasie intestinale gastrique était respectivement de $50 \%$ et de $100 \%$. Dans notre étude, la sensibilité était faible à $19 \%$ et la spécificité très élevée à $100 \%$. Comme dans la plupart des études japonaises, il faut noter que la précédente étude était réalisée en utilisant des endoscopes avec un zoom optique x 80 et avec prise 
d'agents mucolytiques avant l'endoscopie ; ce qui permettait un meilleur rendement des endoscopies et donc une sensibilité plus élevée.

Concernant la formation sur la détection endoscopique de la métaplasie intestinale gastrique par le NBI, le taux de bonnes réponses augmentait de façon statistiquement significative entre la session 1 réalisée avant la formation, et la session 2 après la formation ( $70 \%$ à $80 \%, p=0.035)$.

Une seule autre étude sur ce sujet était retrouvée (24). Il s'agissait d'une étude portugaise qui comportait 200 vidéos réparties sur 20 sessions, analysées par six endoscopistes dont trois juniors qui avaient moins de 4 ans d'expérience en endoscopie, et trois séniors qui avaient au moins 9 ans d'expérience en endoscopie. Les vidéos étaient également réalisées avec des endoscopes à faible zoom digitale x1,5. Les critères étudiés pour la détection de la métaplasie intestinale gastrique étaient l'aspect tubulo-villeux des glandes avec une muqueuse et des vaisseaux réguliers, et les LBC. Lors de cette étude, les endoscopistes devaient tout d'abord faire la formation sur la détection de la métaplasie puis étaient ensuite testés sur les 200 vidéos, il n'existait pas de pré-test pour évaluer les connaissances initiales, avant la formation.

Dans cette étude tout comme dans notre étude, l'expérience en endoscopie n'était pas retrouvée comme étant un avantage. Dans notre étude, le taux de bonne réponses, la sensibilité et la spécificité initiales dans le pré-test avaient une tendance à être plus élevée dans le groupe séniors (respectivement $65 \%, 60 \%$ et $70 \%$ contre $76 \%, 77 \%$ et $74 \%, p>0,05$ ) comme attendu, mais la progression entre la session 1 et la session 3 avait une tendance à être plus élevée dans le groupe junior sur les trois critères de validité, pour terminer avec une tendance à une meilleure sensibilité et un meilleur taux de bonnes réponses que les seniors (respectivement $79 \%$ et $80 \%$ contre $71 \%$ et $76 \%$ ). Ces résultats pouvaient s'expliquer possiblement par une motivation supérieure pour les juniors d'apprendre une nouvelle classification, le désir d'approfondir leurs connaissances médicales en étant au début de leurs carrières, et peut être leur facilité pour envisager une formation informatisée. 
Concernant l'amélioration de la détection de la métaplasie intestinale gastrique dans l'apprentissage par critère, nous observions dans notre étude une augmentation statistiquement significative du taux de bonnes réponses lorsque le critère light blue crest était présent, entre la session 1 et la session 3 (augmentation de 20\%, p=0,013), et entre la session 2 et la session $3(p=0,006)$. L'apprentissage des deux autres critères permettait une augmentation du taux de bonnes réponses mais non significative ( $p>$ $0,05)$.

Ces résultats pouvaient peut être s'expliquer par la complexité et la finesse à détecter des lights blue crest alors que les 2 autres caractères pouvaient sembler plus évidents de premier abord. Cependant en l'absence de magnification, la sensibilité de ce critère restait faible tout comme pour la WOS, et c'était donc le critère tubulo-villeux qui avait la meilleure spécificité et sensibilité pour la détection de la métaplasie intestinale gastrique lors d'une endoscopie digestive haute standard, avec la simple pression sur un bouton qui permettait d'avoir le NBI.

La méthodologie de notre étude était différente de celle présentée par Dias-Silva et al. (24) ; notamment par la session 1 nous réalisions un pré-test permettant d'évaluer les connaissances initiales des observateurs et d'observer l'apport réel de notre formation. Une $3^{\text {ème }}$ session était réalisée, permettant d'augmenter l'entrainement et donc l'expérience des observateurs, et d'objectiver si une correction supplémentaire apportait des informations pertinentes permettant d'augmenter les performances. Seul le taux de bonnes réponses par détection des LBC augmentait de manière statistiquement significative entre les sessions 2 et 3.

II est à noter que trois vidéos avaient un taux de bonnes réponses inférieur à $50 \%$. II s'agissait de la vidéo 6 de la session 2, qui retrouvait de la métaplasie intestinale associée à de la dysplasie de bas grade, la vidéo 1 de la session 3 qui retrouvait une gastrite atrophique marquée associée à de la métaplasie intestinale, et la vidéo 7 de la session 3 qui retrouvait un polype hyperplasique sur terrain de gastrite chronique, associé à de la métaplasie intestinale. 
Lors de la formation, les caractéristiques des muqueuses retrouvant de la dysplasie ou un polype associé à de la métaplasie intestinale n'étaient pas décrites. Peut-être faudrait-il compléter la formation par la description de ces situations endoscopiques moins fréquentes et difficiles à repérer, mais nécessitant probablement d'être étudiées en formation afin de sensibiliser l'œil des endoscopistes.

Notre étude avait également ses limites. Nous ne retrouvions pas d'augmentation significative de la sensibilité et de la spécificité probablement par manque de puissance. Le nombre de vidéos était calculé pour obtenir une augmentation significative de la sensibilité avec une puissance de $80 \%$, il faudrait l'analyse de 680 vidéos alors que 450 vidéos étaient visionnées dans notre étude, réparties sur 15 opérateurs. Cette étude pourrait être ajustée sur 23 opérateurs ou un nombre de vidéos visualisées par opérateurs plus important ; pour voir si cela permettrait une augmentation statistiquement significative de la sensibilité. De plus, la possibilité de réponse était binaire : présence ou absence de métaplasie, ce qui laissait plus de chance au hasard. Si plus de possibilités de réponses avaient été envisagées portant sur les autres types de lésions, ou la présence des critères permettant de détecter la métaplasie ; cela aurait peut-être permis de dégager plus de différences significatives. Plusieurs biais pouvaient apparaitre dans cette étude, notamment le fait que la difficulté des vidéos sélectionnées pour la formation était définie par les auteurs de l'étude, basée sur leurs expériences. II est possible que le nombre de vidéos difficiles et faciles ait été mal réparti au cours des trois sessions du fait d'un biais de sélection. Peut-être l'avis d'autres endoscopistes expérimentés serait utile. De plus, il y avait un taux de métaplasie plus élevé dans la session 3, choisi à cause de la qualité et de la difficulté des vidéos ; ceci pouvait également créer un biais dans les réponses des opérateurs car les vidéos présentant de la métaplasie et l'absence de métaplasie étaient répartie de façon égale dans les deux sessions précédentes. Nous n'avions pas réalisé de retour d’information par les opérateurs concernant l'évaluation de la formation et des tests, comme réalisé dans l'étude de Dias-Silva et al. (24). Ceci permettrait d'adapter la formation aux attentes des opérateurs, d'améliorer la formation et d'obtenir de meilleures performances dans le diagnostic. 


\section{CONCLUSION ET PERSPECTIVES}

La détection de la métaplasie intestinale gastrique reste un enjeu important de la prévention des cancers gastriques, pour dépister les patients nécessitant une surveillance rapprochée afin de détecter au plus tôt l'apparition de dysplasie de bas et haut grade.

Cette étude apporte un premier outil d'apprentissage innovant, simple, afin d'utiliser au mieux le NBI qui a fait ses preuves dans la détection de la métaplasie intestinale gastrique et qui figure actuellement dans les recommandations internationales.

En effet, la large surface de l'estomac et le caractère souvent focal de la métaplasie intestinale font que les biopsies non ciblées peuvent facilement passer à côté de cette lésion. Cibler les biopsies grâce au NBI permettrait une meilleure rentabilité diagnostic.

Par ailleurs, cette étude s'intéressait à la caractérisation des lésions et non à leur détection. Il pourrait être intéressant, dans une future étude, d'évaluer les taux de détection de la métaplasie intestinale gastrique dans les groupes à risque (cancers de l'estomac ou gastrectomie partielle pour cancer, maladie de Biermer et autres gastrites auto-immunes), lors d'une endoscopie digestive haute. 


\section{BIBLIOGRAPHIE}

1. Zaanan A, Bouché O, Benhaim L, Buecher B, Chapelle N, Dubreuil O, et al. Gastric cancer: French intergroup clinical practice guidelines for diagnosis, treatments and follow-up (SNFGE, FFCD, GERCOR, UNICANCER, SFCD, SFED, SFRO). Dig Liver Dis. août 2018;50(8):768-79.

2. Chapelle N, Bouvier A-M, Manfredi S, Drouillard A, Lepage C, Faivre J, et al. Early Gastric Cancer: Trends in Incidence, Management, and Survival in a Well-Defined French Population. Ann Surg Oncol. oct 2016;23(11):3677- 83.

3. Binder-Foucard F, Bossard N, Delafosse P, Belot A, Woronoff A-S, Remontet L. Cancer incidence and mortality in France over the 1980-2012 period: Solid tumors. Rev DÉpidémiologie Santé Publique. avr 2014;62(2):95 - 108.

4. Hu Y, Fang JY, Xiao SD. Can the incidence of gastric cancer be reduced in the new century?: Can the incidence of GC be reduced? J Dig Dis. janv 2013;14(1):11- 5.

5. Correa P. A MODEL FOR GASTRIC CANCER EPIDEMIOLOGY. The Lancet. juill 1975;306(7924):58- 60 .

6. Dinis-Ribeiro M, Areia M, de Vries A, Marcos-Pinto R, Monteiro-Soares M, O'Connor $A$, et al. Management of precancerous conditions and lesions in the stomach (MAPS): guideline from the European Society of Gastrointestinal Endoscopy (ESGE), European Helicobacter Study Group (EHSG), European Society of Pathology (ESP), and the Sociedade Portuguesa de Endoscopia Digestiva (SPED). Endoscopy. janv 2012;44(01):74- 94.

7. Dinis-Ribeiro M. A follow up model for patients with atrophic chronic gastritis and intestinal metaplasia. J Clin Pathol. 1 févr 2004;57(2):177- 82.

8. Uedo N, Ishihara R, lishi H, Yamamoto S, Yamamoto S, Yamada T, et al. A new method of diagnosing gastric intestinal metaplasia: narrow-band imaging with magnifying endoscopy. Endoscopy. août 2006;38(8):819- 24.

9. Uemura N, Okamoto S, Yamamoto S, Matsumura N, Yamaguchi S, Yamakido M, et al. Infection and the Development of Gastric Cancer. N Engl J Med. 13 sept 2001;345:784- 9.

10. Cassaro M, Rugge M, Gutierrez O, Leandro G, Graham DY, Genta RM. Topographic patterns of intestinal metaplasia and gastric cancer. Am J Gastroenterol. juin 2000;95(6):1431- 8.

11. Kuznetsov K, Lambert R, Rey J-F. Narrow-Band Imaging: Potential and Limitations. Endoscopy. janv 2006;38(01):76- 81. 
12. Bhat YM, Abu Dayyeh BK, Chauhan SS, Gottlieb KT, Hwang JH, Komanduri S, et al. High-definition and high-magnification endoscopes. Gastrointest Endosc. déc 2014;80(6):919- 27.

13. Anagnostopoulos G, Yao K, Kaye P, Fogden E, Fortun P, Shonde A, et al. Highresolution magnification endoscopy can reliably identify normal gastric mucosa, Helicobacter pylori -associated gastritis, and gastric atrophy. Endoscopy. mars 2007;39(3):202- 7.

14. Bansal A, Ulusarac O, Mathur S, Sharma P. Correlation between narrow band imaging and nonneoplastic gastric pathology: a pilot feasibility trial. Gastrointest Endosc. févr 2008;67(2):210- 6.

15. Kanemitsu T, Yao K, Nagahama T, Imamura K, Fujiwara S, Ueki T, et al. Extending magnifying NBI diagnosis of intestinal metaplasia in the stomach: the white opaque substance marker. Endoscopy. juin 2017;49(06):529- 35.

16. Vannella L, Lahner E, Osborn J, Cesare B, Massimo M, Delle Fave G, et al. Risk factors for progression to gastric neoplastic lesions in patients with atrophic gastritis. Aliment Pharmacol Ther [Internet]. févr 2010 [cité 14 sept 2018]; Disponible sur: http://doi.wiley.com/10.1111/j.1365-2036.2010.04268.x

17. Whiting JL. The long term results of endoscopic surveillance of premalignant gastric lesions. Gut. 1 mars 2002;50(3):378- 81.

18. de Lange T, Svensen AM, Larsen S, Aabakken L. The functionality and reliability of an Internet interface for assessments of endoscopic still images and video clips: distributed research in gastroenterology. Gastrointest Endosc. mars 2006;63(3):445- 52.

19. Pimentel-Nunes P, Dinis-Ribeiro M, Soares J, Marcos-Pinto R, Santos C, Rolanda C, et al. A multicenter validation of an endoscopic classification with narrow band imaging for gastric precancerous and cancerous lesions. Endoscopy. 31 janv 2012;44(03):236- 46.

20. An JK, Song GA, Kim GH, Park DY, Shin NR, Lee BE, et al. Marginal turbid band and light blue crest, signs observed in magnifying narrow-band imaging endoscopy, are indicative of gastric intestinal metaplasia. 2012;6.

21. Capelle LG, Haringsma J, de Vries AC, Steyerberg EW, Biermann K, van Dekken H, et al. Narrow Band Imaging for the Detection of Gastric Intestinal Metaplasia and Dysplasia During Surveillance Endoscopy. Dig Dis Sci. déc 2010;55(12):3442 - 8.

22. Rerknimitr R. Non-sequential narrow band imaging for targeted biopsy and monitoring of gastric intestinal metaplasia. World J Gastroenterol. 2011;17(10):1336. 
23. Yao K, Iwashita A, Tanabe H, Nishimata N, Nagahama T, Maki S, et al. White opaque substance within superficial elevated gastric neoplasia as visualized by magnification endoscopy with narrow-band imaging: a new optical sign for differentiating between adenoma and carcinoma. Gastrointest Endosc. sept 2008;68(3):574- 80 .

24. Dias-Silva D, Pimentel-Nunes $P$, Magalhães J, Magalhães R, Veloso N, Ferreira C, et al. The learning curve for narrow-band imaging in the diagnosis of precancerous gastric lesions by using Web-based video. Gastrointest Endosc. juin 2014;79(6):910- 20. 


\section{ANNEXES}

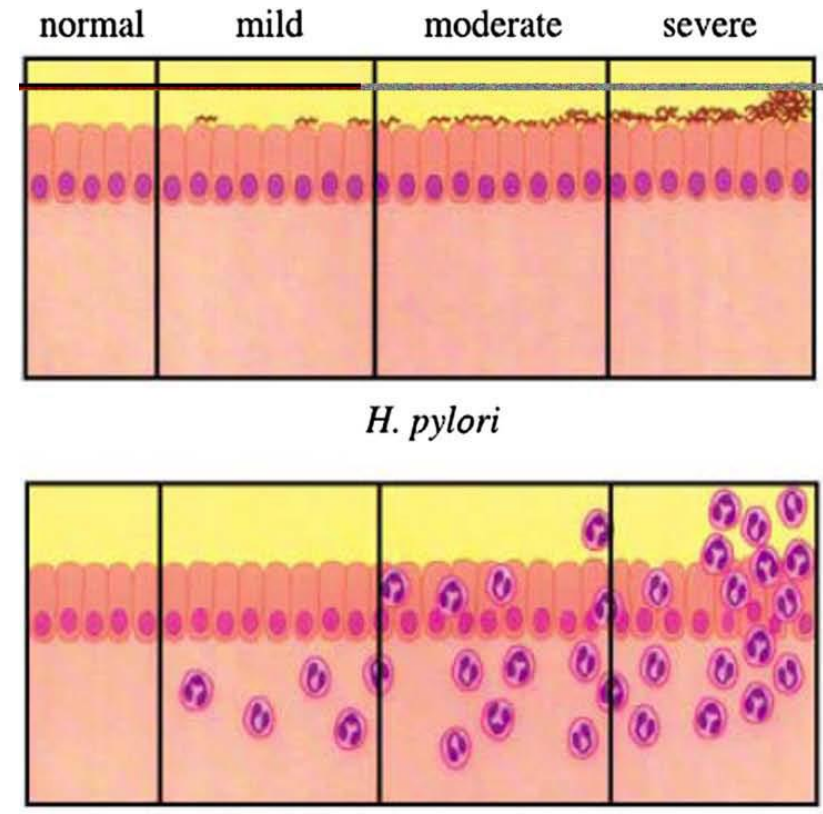

neutrophils

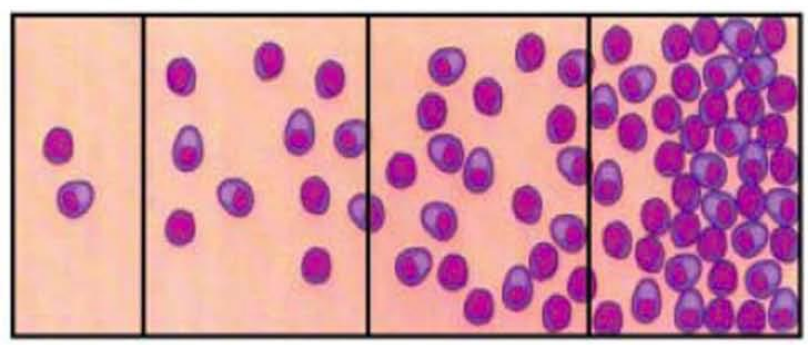

monocytes

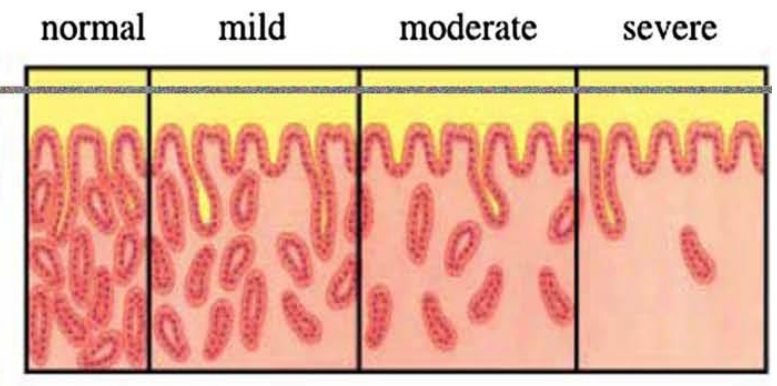

atrophy (antrum)

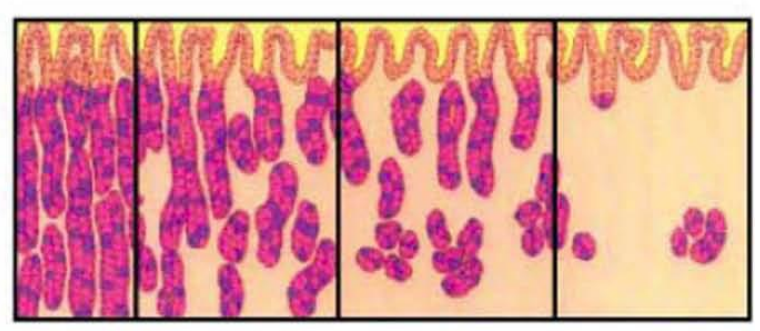

atrophy (corpus)

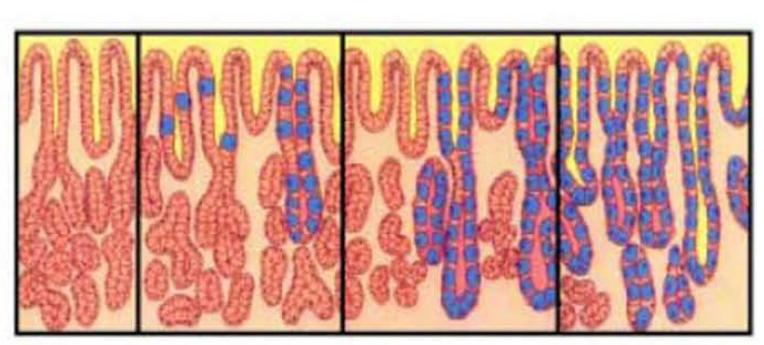

intestinal metaplasia

Annexe 1 : grille d'évaluation de la classification de Sydney 


\begin{tabular}{|c|c|c|}
\hline Type of gastritis & Etiologic factors & Gastritis synonyms \\
\hline Nonatrophic & $\begin{array}{l}\text { Helicobacter pylori } \\
\text { ?Other factors }\end{array}$ & $\begin{array}{l}\text { Superficial } \\
\text { Diffuse antral gastritis (DAG) } \\
\text { Chronic antral gastritis (CAG) } \\
\text { Interstitial-follicular } \\
\text { Hypersecretory } \\
\text { Type } B^{a}\end{array}$ \\
\hline \multicolumn{3}{|l|}{ Atrophic } \\
\hline Autoimmune & Autoimmunity & $\begin{array}{l}\text { Type } A^{a} \\
\text { Diffuse Corporal } \\
\text { Pernicious anemia-associated }\end{array}$ \\
\hline Multifocal atrophic & $\begin{array}{l}\text { Helicobacter pylori } \\
\text { Dietary } \\
\text { ?Environmental factors }\end{array}$ & $\begin{array}{l}\text { Type } \mathrm{B},{ }^{a} \text { type } \mathrm{AB}^{a} \\
\text { Environmental } \\
\text { Metaplastic }\end{array}$ \\
\hline \multicolumn{3}{|l|}{ Special forms } \\
\hline Chemical $^{b}$ & $\begin{array}{l}\text { Chemical irritation } \\
\text { Bile } \\
\text { NSAIDs } \\
\text { ? Other agents }\end{array}$ & $\begin{array}{l}\text { Reactive } \\
\text { Reflux } \\
\text { NSAID } \\
\text { Type } \text { C }^{a}\end{array}$ \\
\hline Radiation & Radiation injury & \\
\hline Lymphocytic & $\begin{array}{l}\text { Idiopathic? Immune mechanisms } \\
\text { Gluten } \\
\text { Drug (ticlopidine) } \\
\text { ? H. pylori }\end{array}$ & $\begin{array}{l}\text { Varioliform (endoscopic) } \\
\text { Celiac disease-associated }\end{array}$ \\
\hline $\begin{array}{l}\text { Noninfectious } \\
\text { granulomatous }\end{array}$ & $\begin{array}{l}\text { Crohn's disease } \\
\text { Sarcoidosis } \\
\text { Wegener's granulomatosis and other vasculitides } \\
\text { Foreign substances }\end{array}$ & \\
\hline & Idiopathic & Isolated granulomatous \\
\hline Eosinophilic & $\begin{array}{l}\text { Food sensitivity } \\
\text { ? Other allergies }\end{array}$ & Allergic \\
\hline Other infectious gastritides & $\begin{array}{l}\text { Bacteria (other than H. pylori) } \\
\text { Vlruses } \\
\text { Fungi } \\
\text { Parasites }\end{array}$ & Phlegmonous \\
\hline
\end{tabular}

NSAIDs, nonsteroidal anti-inflammatory drugs.

${ }^{a}$ Alphabetic designations of gastritis were abandoned in the original presentation of the Sydney System. That approach is also recommended here. Use of "Type B" to denote either atrophic or non-atrophic gastritis is considered to be especially misleading.

${ }^{b}$ Many participants favor substitution of gastropathy for gastritis to describe conditions that result from chemical injury.

\section{Annexe 2: Système de Sidney modifié}




\begin{tabular}{|c|c|c|c|c|c|}
\hline \multicolumn{2}{|c|}{ Score de l'atrophie } & \multicolumn{4}{|c|}{ CORPS GASTRIQUE } \\
\hline & & Absente & $\begin{array}{l}\text { Légère (glandes } \\
\text { atrophiques } \\
<30 \% \text { ) }\end{array}$ & $\begin{array}{l}\text { Modérée (glandes } \\
\text { atrophiques } 30 \%-60 \% \text { ) }\end{array}$ & $\begin{array}{l}\text { Sévère (glandes } \\
\text { atrophiques }>60 \% \text { ) }\end{array}$ \\
\hline \multirow{4}{*}{ ANTRE } & Absente & Classe 0 & Classe I & Classe II & Classe II \\
\hline & $\begin{array}{l}\text { Légère (glandes } \\
\text { atrophiques } \\
<30 \% \text { ) }\end{array}$ & Classe I & Classe I & Classe II & Classe III \\
\hline & $\begin{array}{l}\text { Modérée (glandes } \\
\text { atrophiques 30\%-60\%) }\end{array}$ & Classe II & Classe II & Classe III & Classe IV \\
\hline & $\begin{array}{l}\text { Sévère (glandes } \\
\text { atrophiques > } 60 \% \text { ) }\end{array}$ & Classe III & Classe III & Classe IV & Classe IV \\
\hline
\end{tabular}

Annexe 3 : systèmes de classification OLGA

\begin{tabular}{|c|c|c|c|c|c|}
\hline \multicolumn{2}{|c|}{ Score de la métaplasie intestinale } & \multicolumn{4}{|c|}{ CORPS GASTRIQUE } \\
\hline & & Absente & $\begin{array}{l}\text { Légère (glandes en MI } \\
<30 \%)\end{array}$ & $\begin{array}{l}\text { Modérée } \\
\text { (glandes en MI } \\
30 \%-60 \% \text { ) }\end{array}$ & $\begin{array}{l}\text { Sévère } \\
\text { (glandes en MI } \\
>60 \% \text { ) }\end{array}$ \\
\hline \multirow{4}{*}{ ANTRE } & Absente & Classe 0 & Classe I & Classe II & Classe II \\
\hline & $\begin{array}{l}\text { Légère (glandes en MI } \\
<30 \% \text { ) }\end{array}$ & Classe I & Classe I & Classe II & Classe III \\
\hline & $\begin{array}{l}\text { Modérée (glandes en MI } \\
30 \%-60 \% \text { ) }\end{array}$ & Classe II & Classe II & Classe III & Classe IV \\
\hline & $\begin{array}{l}\text { Sévère (glandes en MI } \\
>60 \% \text { ) }\end{array}$ & Classe III & Classe III & Classe IV & Classe IV \\
\hline
\end{tabular}

Annexe 4 : Système de classification OLGIM : Operative Link on Intestinal Metaplasia 


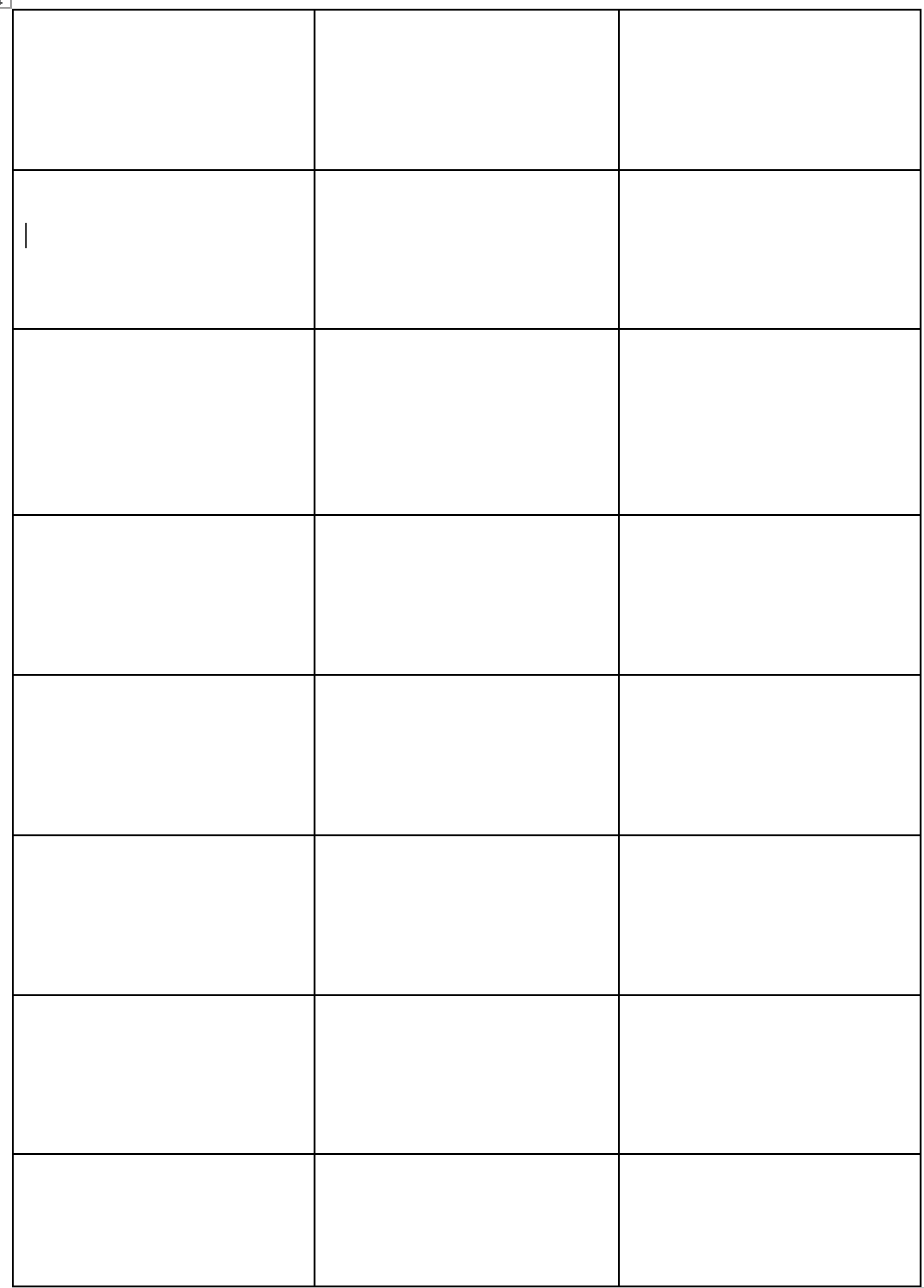

Annexe 5 : tableau de recueil des patients 


\begin{tabular}{|l|l|l|l|l|}
\hline $\begin{array}{l}N^{\circ} \\
\text { questions }\end{array}$ & \multicolumn{2}{|l|}{ Métaplasie intestinale } & \multicolumn{2}{l|}{ Degrés de certitude } \\
\hline & OUI & NON & FAIBLE & ÉLEVÉ \\
\hline 1 & & & & \\
\hline 2 & & & & \\
\hline 3 & & & & \\
\hline 4 & & & & \\
\hline 5 & & & & \\
\hline 6 & & & & \\
\hline 7 & & & & \\
\hline 8 & & & & \\
\hline 10 & & & & \\
\hline
\end{tabular}

Annexe 6: fiche de recueil des réponses des observateurs 


\section{SERMENT}

En présence des Maîtres de cette école, de mes chers condisciples et devant l'effigie d'Hippocrate, je promets et je jure, au nom de l'Etre suprême, d'être fidèle aux lois de l'honneur et de la probité dans lexercice de la médecine.

$>$ Je donnerai mes soins gratuits à Cindigent et n'exigerai jamais un salaire au-dessus de mon travail.

Admis (e) dans lintérieur des maisons, mes yeux ne verront pas ce qui s'y passe, ma langue taira les secrets qui me seront confiés, et mon état ne servira pas à corrompre les mours, ni à favoriser le crime.

$>$ Respectueux (se) et reconnaissant (e) envers mes Maîtres, je rendrai à leurs enfants ['instruction que j'ai reçue de leurs pères.

Que les hommes m'accordent leur estime si je suis fidèle à mes promesses. Que je sois couvert (e) d'opprobre et méprisé (e) de mes confrères si j'y manque. 


\section{RÉSUMÉ :}

Introduction: L'atrophie gastrique et la métaplasie intestinale gastrique sont des lésions précancéreuses identifiées depuis longtemps. La chromo-endoscopie électronique telle que le Narrow-Band Imaging (NBI), simple d'utilisation, a montré une supériorité de détection de ces lésions par rapport à la lumière blanche ; mais elle n'est cependant pas utilisée en routine en Europe. Il est probable que cela soit dû au manque de formation des gastroentérologues. L'objectif de cette étude était d'évaluer la performance d'un programme d'enseignement en ligne pour la détection de la métaplasie intestinale gastrique dans les conditions d'endoscopie standard en France, chez des gastroentérologues avec différents niveaux de formation.

Matériels et méthodes: L'étude comportait 1) une session avant enseignement (session 1 ou prétest) avec 10 vidéos d'endoscopie gastrique avec et sans NBI, 2) un diaporama sonorisé présentant l'aspect de la muqueuse gastrique avec et sans NBI et les critères conduisant au diagnostic de métaplasie intestinale, 3) deux sessions de post test de 10 vidéos chacune. Ces 30 vidéos d'endoscopie gastrique étaient présentées à 15 gastroentérologues : 8 juniors ( $\leq 6$ ans d'expérience en endoscopie) et 7 séniors ( $>6$ ans). Ils donnaient leur diagnostic accompagné d'un degré de confiance. Les réponses des lecteurs étaient comparées aux résultats anatomo-pathologiques. Une correction détaillée de chaque vidéo était transmise après chaque session.

Résultats : Trois critères morphologiques permettaient la détection endoscopique de la métaplasie intestinale: aspect tubulovilleux (Se 76\% Sp 83\% VPP 78\% VPN 82\%), présence de crêtes bleu-clair (21\%-94\%-75\%-61\%) et de substance blanche opaque (19\%-100\%-100\%-62\%). Le taux de bonnes réponses augmentait significativement entre la session 1 et $2(p<0,05)$. Il passait de $65 \%$ à $80 \%$ pour les juniors et de 76 à $80 \%$ pour les séniors (NS). Il existait également une augmentation significative du taux de bonnes réponses lorsque le critère crêtes bleu-claires était présent $(73 \%$ à $93 \% \mathrm{p}<0,05)$. II y avait plus de vidéos avec un taux de bonnes réponses $>85 \%$ à la dernière session $(6 / 10)$ qu'à la session prétest (3/10).

Conclusion: La caractérisation endoscopique de la métaplasie intestinale n'est pas seulement accessible aux gastroentérologues asiatiques maitrisant l'endoscopie à fort grossissement chez des patients souvent préparés avec spasmolytiques et mucolytiques. Elle peut être améliorée avec un matériel d'endoscopie standard par une formation adéquate accessible aux gastroentérologues confirmés ou novices. Cette étude montre qu'avec une formation comprenant un diaporama et 30 vidéos didactiques nous pouvons améliorer nos performances dans le diagnostic de métaplasie intestinale gastrique.

MOTS CLEFS : endoscopie, métaplasie intestinale gastrique, NBI, programme d'enseignement, vidéos, tubulovilleux, crêtes bleu claires, substance blanche opaque. 